\title{
Algebraic Analysis of Demodalised Analytic Implication
}

\author{
Antonio Ledda ${ }^{1,2} \cdot$ Francesco Paoli $^{1} \cdot$ Michele Pra Baldi $^{1}$
}

Received: 26 September 2018 / Accepted: 18 January 2019 / Published online: 13 February 2019

(C) Springer Nature B.V. 2019

\begin{abstract}
The logic DAI of demodalised analytic implication has been introduced by J.M. Dunn (and independently investigated by R.D. Epstein) as a variation on a timehonoured logical system by C.I. Lewis' student W.T. Parry. The main tenet underlying this logic is that no implication can be valid unless its consequent is "analytically contained" in its antecedent. DAI has been investigated both proof-theoretically and model-theoretically, but no study so far has focussed on DAI from the viewpoint of abstract algebraic logic. We provide several different algebraic semantics for DAI, showing their equivalence with the known semantics by Dunn and Epstein. We also show that DAI is algebraisable and we identify its equivalent quasivariety semantics. This class turns out to be a linguistic and axiomatic expansion of involutive bisemilattices, a subquasivariety of which forms the algebraic counterpart of Paraconsistent Weak Kleene logic (PWK). This fact sheds further light on the relationship between containment logics and logics of nonsense.
\end{abstract}

Keywords Analytic implication - Dependence logic - Abstract algebraic logic . Płonka sums · Regular varieties · Relevance logic

\section{Introduction}

Containment logics are a family of logics based on the idea that a necessary condition for an argument to be valid is that its conclusion be "analytically contained" in

Antonio Ledda

antonio.ledda@unica.it; antonio.ledda@vanderbilt.edu

Francesco Paoli

paoli@unica.it

Michele Pra Baldi

m.prabaldi@gmail.com

1 Dipartimento di Pedagogia, Psicologia, Filosofia, Università di Cagliari, Cagliari, Italy

2 Department of Mathematics, Vanderbilt University, Nashville TN, USA 
its premisses - or on the related idea that a necessary condition for an implication to be valid is that its consequent be "analytically contained" in its antecedent. What exactly counts as analytic containment is left to the specifics of the particular logic at issue; for propositional logics, it usually amounts to an inclusion constraint among the sets of propositional variables occurring in the sentences under consideration. Here, we will be concerned with logics L (with a binary connective $\rightarrow$ in their language) that satisfy what Ferguson [13] calls the proscriptive principle for theorems $(\mathbf{P P} \rightarrow)$ : letting $\operatorname{Var}(\chi)$ be the set of propositional variables in $\chi$ for any formula $\chi$, $\mathbf{P P}^{\rightarrow}$ requires that if $\vdash_{\mathrm{L}} \varphi \rightarrow \psi$, then $\operatorname{Var}(\psi) \subseteq \operatorname{Var}(\varphi) . \mathbf{P P}^{\rightarrow}$ can be motivated in various ways [12], for instance as a relevance constraint-indeed, a tighter one than the customary variable-sharing requirement of relevance logics.

Historically, the first logical system obeying $\mathbf{P P} \rightarrow$ was PAI, the logic of analytic implication introduced in the early 1930's [19], and modified in later writings [20], by C.I. Lewis' student W.T. Parry. Although Parry's approach never became mainstream within relevance logics, it drew the attention of notable scholars over the following decades (see e.g. [8, 14, 17, 27]). In particular, Kit Fine provided PAI with a double-barrelled analysis in the sense of Sylvan [26], revealing that PAI is ultimately obtained from imposing a "linguistic strainer" on the modal logic S5. Observe, indeed, that although the language of PAI is the same as for classical logic (hereafter sometimes noted CL), a necessity connective can be introduced as usual in relevance logics via $\square \varphi=(\varphi \rightarrow \varphi) \rightarrow \varphi$. For further information on PAI and its history, see the comprehensive monograph [13].

In 1972, J. Michael Dunn [8] explored what happens if we "demodalise" PAI by adding to it the axiom of collapse of modality $\varphi \rightarrow \square \varphi$. The resulting logic DAI of demodalised analytic implication turns out to be much smoother, and to have more interesting formal properties, than Parry's original system. As a relevance logic, DAI is in the same ballpark as $\mathbf{R}$ with Boolean negation [18] or Lewis' systems of strict implication, to the extent that all these logics are fully classical in their $\rightarrow$-free fragment. Later on, DAI was independently rediscovered by R.D. Epstein under the heading of dependence logic [10].

From the proof-theoretic viewpoint, DAI has been endowed with Hilbert-style calculi [8, 10], tableaux calculi [5], and sequent calculi [11]. Model-theoretically, it has been analysed both with the standard methods of possible-world semantics [8] and via more unusual semantics especially tailored by Epstein for the needs of containment logics and their neighbours (see [10] and Section 2.4 below). To the best of our knowledge, the only investigation into the algebraic semantics of DAI is [9], a paper that-also for historical reasons-does not use the concepts and tools of contemporary abstract algebraic logic (AAL). The goal of the present paper is twofold. On the one hand, we aim at filling this gap and study DAI within the framework of AAL. On the other hand, we try to shed further light on the relationships between containment logics and logics of nonsense, another family of logics usually suggested as tools for reasoning in the presence of "nonsensical" propositions (ill-formed sentences, corrupted data, and the like: see e.g. [13]). These connections have been clarified in a number of recent papers $[2,6,7]$; here, we underscore the role played in the algebraic analysis of DAI by involutive bisemilattices, a subquasivariety of which was already 
recognised in [1] as the "algebraic counterpart" (in a sense to be specified below) of Paraconsistent Weak Kleene (PWK), a typical logic of nonsense.

The paper is structured as follows. In Section 2 we dispatch the necessary preliminaries on AAL, on involutive bisemilattices, on an important algebraic construction (Płonka sums) that will take centre stage in what follows, and on demodalised analytic implication. In Section 3 we introduce the quasivariety $\mathcal{I} \mathcal{I B S} \mathcal{L}$ of implicative involutive bisemilattices, expansions of involutive bisemilattices by an additional operation of implication. Notable examples of implicative involutive bisemilattices are $P$-implicative involutive bisemilattices, the algebra reducts of certain matrices that are obtained through a variation on the technique of Płonka sums of matrices. We prove that $\mathrm{P}$-implicative involutive bisemilattices form an algebraic semantics for DAI; this semantics is shown to be equivalent both to the known semantics for our logic (Epstein's set-assignment semantics, Dunn's semantics) and to a new semantics based on certain "twist products" of Boolean algebras and semilattices with zero. In Section 4 we identify the Leibniz reduced models of DAI that are obtained as Płonka sums over appropriate systems of matrices. Finally, in Section 5, we prove that DAI is algebraisable with $\mathcal{I I B S} \mathcal{L}$ as equivalent quasivariety semantics and, as a consequence, we refine the results in the preceding section to a full characterisation of the Leibniz reduced models of DAI and their algebra reducts.

\section{Preliminaries}

\subsection{Abstract Algebraic Logic}

For unexplained terminology and notation on universal algebra and AAL, we refer the reader respectively to [3] and [15]. We denote algebras by boldface capital letters and their universes by italicised capital letters. Given a class of algebras $\mathcal{K}$, we respectively denote by $I(\mathcal{K}), S(\mathcal{K}), P(\mathcal{K})$, and $P_{U}(\mathcal{K})$ the classes of isomorphic images, subalgebras, products, and ultraproducts of algebras in $\mathcal{K} ; V(\mathcal{K})$ is the variety generated by $\mathcal{K} . \mathbf{B}_{n}$ will denote the finite Boolean algebra with $n$ elements, for any positive integer $n$ of the form $2^{k}$. The algebra of formulas of the similarity type $\mathcal{L}(\mathcal{L}$ formulas), over a countably infinite set $\operatorname{Var}(\mathcal{L})$ of generators, is denoted by $\operatorname{Fm}(\mathcal{L})$. Equations of type $\mathcal{L}(\mathcal{L}$-equations) are ordered pairs of $\mathcal{L}$-formulas of the same type, noted $\varphi \approx \psi$. Given $\varphi \in F m(\mathcal{L})$, we denote by $\operatorname{Var}(\varphi)$ the set of variables occurring in $\varphi$. Similarly, given $\Gamma \subseteq F m(\mathcal{L})$, we set $\operatorname{Var}(\Gamma)=\bigcup\{\operatorname{Var}(\gamma): \gamma \in \Gamma\}$. Two distinguished similarity types will play a crucial role in what follows. The former one is the $\langle 2,2,1,0,0\rangle$-type of classical logic and Boolean algebras, with primitive operation symbols $\wedge, \vee, \neg, 0$, and 1 ; this type will be noted $\mathcal{L}_{0}$. The latter one, hereafter referred to as $\mathcal{L}_{1}$, is the expansion of $\mathcal{L}_{0}$ by an additional binary operation symbol $\rightarrow$.

A logic of type $\mathcal{L}$ is an ordered pair $\mathrm{L}=\left\langle\mathbf{F m}(\mathcal{L}), \vdash_{\mathrm{L}}\right\rangle$, in which $\vdash_{\mathrm{L}} \subseteq$ $\mathcal{P}(F m(\mathcal{L})) \times F m(\mathcal{L})$ is a consequence relation that is substitution-invariant, meaning that for every $\sigma \in \operatorname{End}(\mathbf{F m}(\mathcal{L}))$ and for every $\Gamma \cup\{\varphi\} \subseteq F m(\mathcal{L})$, if $\Gamma \vdash \varphi$, then $\sigma[\Gamma] \vdash \sigma(\varphi)$. A logic L of type $\mathcal{L}$ is finitary when the following holds for all $\Gamma \cup\{\varphi\} \subseteq F m(\mathcal{L})$ : if $\Gamma \vdash_{\mathrm{L}} \varphi$, then there is a finite $\Delta \subseteq \Gamma$ s.t. $\Delta \vdash_{\mathrm{L}} \varphi$. Given 
$\varphi, \psi \in F m(\mathcal{L})$, we write $\varphi-\vdash_{\mathrm{L}} \psi$ as shorthand for $\varphi \vdash_{\mathrm{L}} \psi$ and $\psi \vdash_{\mathrm{L}} \varphi$. Given $\Gamma, \Delta \subseteq F m(\mathcal{L})$, we also write $\Gamma \vdash_{\mathrm{L}} \Delta$ as shorthand for: $\Gamma \vdash_{\mathrm{L}} \varphi$, for all $\varphi \in \Delta$.

If $\mathcal{K}$ is a class of algebras, all of the same type $\mathcal{L}$, the equational consequence relation of $\mathcal{K}$ is the relation $\vdash_{E q(\mathcal{K})} \subseteq \wp\left((F m(\mathcal{L}))^{2}\right) \times(F m(\mathcal{L}))^{2}$ such that, for all $E \subseteq(F m(\mathcal{L}))^{2}$ and for all $\varphi \approx \psi \in(F m(\mathcal{L}))^{2}$,

$$
\begin{aligned}
E \vdash_{E q(\mathcal{K})} \varphi \approx \psi \text { iff } & \text { for every } \mathbf{A} \in \mathcal{K} \text { and every } h \in \operatorname{Hom}(\mathbf{F m}(\mathcal{L}), \mathbf{A}), \\
& \text { if } h(\alpha)=h(\beta) \text { for all } \alpha \approx \beta \in E, \text { then } h(\varphi)=h(\psi) .
\end{aligned}
$$

We apply to equational consequence relations the same notational conventions that are in force for logics and their attendant consequence relations.

A matrix of type $\mathcal{L}$ is an ordered pair $\langle\mathbf{A}, F\rangle$ where $\mathbf{A}$ is an algebra of type $\mathcal{L}$ and $F \subseteq A$. In this case, $\mathbf{A}$ is called the algebra reduct of $\langle\mathbf{A}, F\rangle$. The class operators $I, S, P, P_{U}$, previously introduced for algebras, will also be applied to matrices, viewed as first-order structures. Every matrix $\langle\mathbf{A}, F\rangle$ of type $\mathcal{L}$ induces a logic of the same type whose consequence relation is determined as follows:

$$
\begin{aligned}
\Gamma \vdash_{\langle\mathbf{A}, F\rangle} \varphi \text { iff } & \text { for every } h \in \operatorname{Hom}(\boldsymbol{F m}(\mathcal{L}), \mathbf{A}), \\
& \text { if } h[\Gamma] \subseteq F, \text { then } h(\varphi) \in F .
\end{aligned}
$$

For $\mathrm{M}$ a class of matrices, we write $\Gamma \vdash_{\mathrm{M}} \varphi$ to mean $\Gamma \vdash_{\langle\mathbf{A}, F\rangle} \varphi$ for every $\langle\mathbf{A}, F\rangle \in \mathrm{M}$. A matrix $\langle\mathbf{A}, F\rangle$ is a model of the logic $\mathrm{L}$, both of type $\mathcal{L}$, when $\vdash_{\mathrm{L}} \subseteq_{\langle\mathbf{A}, F\rangle}$; a logic $\mathrm{L}$ is complete w.r.t. a class of matrices $\mathrm{M}$ when $\vdash_{\mathrm{L}}=\vdash_{\mathrm{M}}$. A set $F \subseteq A$ is a deductive filter of $\mathrm{L}$ (or L-filter) on the algebra $\mathbf{A}$, when the matrix $\langle\mathbf{A}, F\rangle$ is a model of $\mathrm{L}$.

Let $\mathbf{A}$ be an algebra and $F \subseteq A$. A congruence $\theta$ of $\mathbf{A}$ is compatible with $F$ when $F$ is a union of $\theta$-cosets. The largest congruence of $\mathbf{A}$ that is compatible with $F$ always exists; this congruence is called the Leibniz congruence of $F$ on $\mathbf{A}$, and is denoted by $\Omega^{\mathbf{A}} F$. Recall that, given an algebra $\mathbf{A}$, a function $p: A^{n} \rightarrow A$ is a polynomial function of $\mathbf{A}$ if there are a natural number $m$, a formula $\varphi\left(x_{1}, \ldots, x_{n+m}\right)$, and elements $b_{1}, \ldots, b_{m} \in A$ such that

$$
p\left(a_{1}, \ldots, a_{n}\right)=\varphi^{\mathbf{A}}\left(a_{1}, \ldots, a_{n}, b_{1}, \ldots, b_{m}\right)
$$

for every $a_{1}, \ldots, a_{n} \in A$. The next lemma characterises Leibniz congruences:

Lemma 1 [15, Thm. 4.23] Let $\mathbf{A}$ be an algebra, $F \subseteq A$, and $a, b \in A$.

$$
\begin{array}{r}
\langle a, b\rangle \in \Omega^{\mathbf{A}} F \Longleftrightarrow \text { for every unary pol. function } p \text { of } \mathbf{A} \\
\qquad p(a) \in F \text { if and only if } p(b) \in F .
\end{array}
$$

By means of the Leibniz congruence, we can associate to logics two distinguished classes of models. More precisely, given a logic $\mathrm{L}$ of type $\mathcal{L}$, we set

$$
\begin{aligned}
\operatorname{Mod}(\mathrm{L}) & =\{\langle\mathbf{A}, F\rangle:\langle\mathbf{A}, F\rangle \text { is a model of } \mathrm{L}\} ; \\
\operatorname{Mod}^{*}(\mathrm{~L}) & =\left\{\langle\mathbf{A}, F\rangle \in \operatorname{Mod}(\mathrm{L}): \Omega^{\mathbf{A}} F=i d^{\mathbf{A}}\right\} .
\end{aligned}
$$

The above classes of matrices are called, respectively, the classes of models and Leibniz reduced models of L.

Let $\mathrm{L}=\left\langle\mathbf{F m}(\mathcal{L}), \vdash_{\mathrm{L}}\right\rangle$ be a logic of type $\mathcal{L}$, and let $\tau=\left\{\gamma_{i}(x) \approx \delta_{i}(x)\right\}_{i \in I}$ be a set of $\mathcal{L}$-equations in a single variable. We may also view $\tau$ as a function which maps 
formulas in $F m(\mathcal{L})$ to sets of equations of the same type. Thus, we let $\tau(\varphi)$ stand for the set

$$
\left\{\gamma_{i}(x / \varphi) \approx \delta_{i}(x / \varphi)\right\}_{i \in I},
$$

where $\gamma_{i}(x / \varphi)$ refers to the result of uniformly replacing any occurrences of $x$ in $\gamma_{i}$ by $\varphi$, and similarly for $\delta_{i}(x / \varphi)$. For $\Gamma \subseteq F m(\mathcal{L}), \tau(\Gamma)$ is defined as $\bigcup\{\tau(\gamma): \gamma \in \Gamma\}$.

Now, let $\mathcal{K}$ be a class of algebras also of type $\mathcal{L}$. We say that $\mathcal{K}$ is an algebraic semantics for $\mathrm{L}$ if, for some such $\tau$, the following condition holds for all $\Gamma \cup\{\varphi\} \subseteq$ $\operatorname{Fm}(\mathcal{L})$ :

$$
\Gamma \vdash_{\mathrm{L}} \varphi \quad \text { iff } \quad \tau(\Gamma) \vdash_{E q(\mathcal{K})} \tau(\varphi) .
$$

Given an $\mathcal{L}$-equation $\varphi \approx \psi$ and a set of $\mathcal{L}$-formulas in two variables $\rho=$ $\left\{\alpha_{j}(x, y)\right\}_{j \in J}$, we use the abbreviation

$$
\rho(\varphi, \psi)=\left\{\alpha_{j}(x / \varphi, y / \psi)\right\}_{j \in J} .
$$

$\rho$, in the same guise as $\tau$, will be also regarded as a function, mapping this time equations to sets of formulas. Again, for $E \subseteq(F m(\mathcal{L}))^{2}, \rho(E)$ is defined as $\bigcup\{\rho(\varphi, \psi): \varphi \approx \psi \in E\}$

A $\operatorname{logic} \mathrm{L}=\left\langle\mathbf{F m}(\mathcal{L}), \vdash_{\mathrm{L}}\right\rangle$ is said to be algebraisable with equivalent algebraic semantics $\mathcal{K}$ (where $\mathcal{K}$ is a class of algebras of type $\mathcal{L}$ ) iff there exist a map $\tau: F m(\mathcal{L}) \rightarrow \wp\left((F m(\mathcal{L}))^{2}\right)$, and a map $\rho:(F m(\mathcal{L}))^{2} \rightarrow \wp(F m(\mathcal{L}))$, both commuting with substitutions, such that the following conditions hold for any $\Gamma \cup\{\varphi, \psi\} \subseteq F m l_{L}$ and for all $E \subseteq\left(F m l_{L}\right)^{2}$ :

AL1: $\quad \Gamma \vdash_{\mathrm{L}} \varphi$ iff $\tau(\Gamma) \vdash_{E q(\mathcal{K})} \tau(\varphi)$;

AL2: $\quad E \vdash_{E q(\mathcal{K})} \varphi \approx \psi$ iff $\rho(E) \vdash_{\mathrm{L}} \rho(\varphi, \psi)$;

AL3: $\varphi \dashv \vdash_{\mathrm{L}} \rho(\tau(\varphi))$;

AL4: $\varphi \approx \psi \vdash_{E q(\mathcal{K})} \tau(\rho(\varphi, \psi))$.

This definition can be drastically simplified:

Lemma 2 [15, Prop. 3.12] A logic L is algebraisable with equivalent algebraic semantics $\mathcal{K}$ iff it satisfies either AL1 and ALA, or else AL2 and AL3.

Theorem 3 [15, Cor. 3.18] In case L is finitary, any two equivalent algebraic semantics for $\mathrm{L}$ generate the same quasivariety, also called the equivalent quasivariety semantics of $\mathrm{L}$.

Some notions other than algebraisability are of importance in AAL. A logic L of type $\mathcal{L}$ is:

- protoalgebraic, if there is a set of $\mathcal{L}$-formulas $\Delta(x, y)$ in two variables such that $\emptyset \vdash_{\mathrm{L}} \Delta(x, x)$ and $x, \Delta(x, y) \vdash_{\mathrm{L}} y$;

- equivalential, if there is a set of $\mathcal{L}$-formulas $\Delta(x, y)$ in two variables, called congruence formulas for $\mathrm{L}$, such that for every $\langle\mathbf{A}, F\rangle \in \operatorname{Mod}(\mathrm{L})$,

$$
\langle a, b\rangle \in \Omega^{\mathbf{A}} F \text { iff } \Delta^{\mathbf{A}}(a, b) \subseteq F \text { for all } a, b \in A .
$$


- truth-equational, if there is a set of equations $\tau(x)$ in a single variable, called defining equations for $\mathrm{L}$, such that for all $\langle\mathbf{A}, F\rangle \in \operatorname{Mod}^{*}(\mathrm{~L}), a \in F$ iff $\mathbf{A} \vDash$ $\tau^{\mathbf{A}}(a)$, for all $a \in A$;

- $\quad$ selfextensional, if the interderivability relation $\dashv_{\mathrm{L}}$ is a congruence on $\mathbf{F m}(\mathcal{L})$.

Remarkably, if L is equivalential, then $\operatorname{Mod}^{*}(\mathrm{~L})$ is closed under the operators $S$ and $P$. Moreover, every equivalential logic is protoalgebraic. Also, it is not hard to show that a logic is algebraisable iff it is both equivalential and truth-equational $[15, \S 6.4]$.

A matrix is trivial if it is of the form $\langle\mathbf{A}, A\rangle$. Observe that the trivial matrix $\langle\mathbf{1},\{1\}\rangle$ over the trivial algebra is a Leibniz reduced model of every logic. Moreover, if $\mathrm{L}$ is a protoalgebraic logic and $\langle\mathbf{A}, A\rangle \in \operatorname{Mod}^{*}(\mathrm{~L})$ is a trivial matrix, then $\langle\mathbf{A}, A\rangle=\langle\mathbf{1},\{1\}\rangle$. Given a protoalgebraic logic L, we set

$$
\mathrm{A} \lg (\mathrm{L})=\left\{\mathbf{A}: \text { there is } F \subseteq A \text { s.t. }\langle\mathbf{A}, F\rangle \in \operatorname{Mod}^{*}(\mathrm{~L})\right\} .
$$

In other words, $A \lg (\mathrm{L})$ is the class of algebra reducts of matrices in $\operatorname{Mod}^{*}(\mathrm{~L})$. The class $A \lg (\mathrm{L})$ is called the algebraic counterpart of L. For the vast majority of logics $\mathrm{L}$, the class $A \lg (\mathrm{L})$ is the class of algebras intuitively associated with $\mathrm{L}$.

Lemma 4 [15, Lm. 5.78] Let L be a logic defined by a class of matrices M. Then $\mathrm{A} \lg (\mathrm{L}) \subseteq V(\mathcal{K})$, where $\mathcal{K}$ is the class of algebra reducts of matrices in $\mathrm{M}$.

Lemma 5 Let $\mathrm{L}$ be a logic of type $\mathcal{L}$, and let $\epsilon, \delta \in F m(\mathcal{L})$. The following are equivalent:

(1) $\mathrm{A} \lg (\mathrm{L}) \vDash \epsilon \approx \delta$;

(2) $\varphi(\epsilon, \vec{z}) \dashv \vdash_{\mathrm{L}} \varphi(\delta, \vec{z})$, for every $\mathcal{L}$-formula $\varphi(v, \vec{z})$.

Proof See [15, Lm. 5.74(1)] and [15, Thm. 5.76].

\subsection{Plonka Sums and Regularisations of Varieties}

Płonka sums (for which see e.g. [22, 23, 25]) are a useful construction in universal algebra. They are especially designed for the investigation of varieties satisfying only regular identities. We recap hereafter the main definitions and concepts concerning them.

Definition 6 Let $\left\{\mathbf{A}_{i}\right\}_{i \in I}$ be a family of algebras of type $\mathcal{L}$. The triple

$$
\mathfrak{T}=\left\langle\left\{\mathbf{A}_{i}\right\}_{i \in I},\langle I, \leq\rangle,\left\{f_{i, j}: i, j \in I \text { and } i \leq j\right\}\right\rangle
$$

is a direct system of type $\mathcal{L}$ in case:

(1) $\langle I, \leq\rangle$ is a join semilattice;

(2) If $i, j \in I$ and $i \neq j$, then $\mathbf{A}_{i} \cap \mathbf{A}_{j}=\emptyset$;

(3) Each $f_{i, j}$ is a homomorphism from $\mathbf{A}_{i}$ to $\mathbf{A}_{j}$ s.t. $f_{i, i}(x)=x$ and $f_{j, k}\left(f_{i, j}(x)\right)=f_{i, k}(x)$. 
Definition 7 If $\mathfrak{T}=\left\langle\left\{\mathbf{A}_{i}\right\}_{i \in I},\langle I, \leq\rangle,\left\{f_{i, j}: i, j \in I\right.\right.$ and $\left.\left.i \leq j\right\}\right\rangle$ is a direct system of type $\mathcal{L}$, then $\mathbf{P}(\mathfrak{T})=\left\langle\bigcup\left\{\mathbf{A}_{i}\right\}_{i \in I},\left\{g^{n}: g^{n} \in \mathcal{L}\right\}\right\rangle$, where for $a_{p} \in \mathbf{A}_{i_{p}}(p \leq n)$ and $k=i_{1} \vee \ldots \vee i_{n}$,

$$
g^{\mathbf{P}(\mathfrak{T})}\left(a_{1}, \ldots, a_{n}\right)=g^{\mathbf{A}_{k}}\left(f_{i_{1}, k}\left(a_{1}\right), \ldots, f_{i_{n}, k}\left(a_{n}\right)\right),
$$

is a total algebra also of type $\mathcal{L}$, called the Płonka sum over $\mathfrak{T}$.

If $\mathcal{L}$ has constants, then the previous definitions are enriched by the stipulations that $\langle I, \leq\rangle$ is a join semilattice with zero whose bottom element is $\perp$, and that $c^{\mathbf{P}}=$ $c^{\mathbf{A}_{\perp}}$ for all constants $c$ in $\mathcal{L}$.

It turns out that, for an algebra of a given type, representability as a Płonka sum over a direct system depends on the presence of certain functions, called partition functions.

Definition 8 Let $\mathbf{A}=\left\langle A,\left\{g^{n}: g^{n} \in \mathcal{L}\right\}\right\rangle$ be an algebra of type $\mathcal{L}$. A function $p$ : $A^{2} \rightarrow A$ is a partition function in $\mathbf{A}$ if the following conditions are satisfied for all $a, b, c, a_{i} \in A$ and for all $g^{n} \in \mathcal{L}$ :

P1: $\quad p(a, a)=a$;

P2: $\quad p(a, p(b, c))=p(a, p(c, b))=p(p(a, b), c)$;

P3: $\quad p\left(g^{n}(\vec{a}), b\right)=g^{n}\left(p\left(a_{1}, b\right), \ldots, p\left(a_{n,}, b\right)\right)$;

P4: $\quad p\left(b, g^{n}(\vec{a})\right)=p\left(b, g^{n}\left(p\left(b, a_{1},\right), \ldots, p\left(b, a_{n},\right)\right)\right)$;

P5: $\quad g^{n}(\vec{a})=p\left(g^{n}(\vec{a}), a_{i}\right)$ for $1 \leq i \leq n$;

P6: $p\left(a, g^{n}(a, \ldots, a)\right)=a$.

Any algebra $\mathbf{A}$ admitting a partition function $p$ can be partitioned into classes $\left\{A_{i}: i \in I\right\}$, where $a, b \in A$ belong to the same component $A_{i}$ exactly when $a=p(a, b)$ and $b=p(b, a)$. Moreover, every $A_{i}$ is the universe of a subalgebra $\mathbf{A}_{i}$ of $\mathbf{A}$. The relation $\leq$ on $I$ given by the rule

$$
i \leq j \text { iff there exist } a \in A_{i}, b \in A_{j} \text { such that } p(b, a)=b
$$

is a semilattice order. Moreover, for all $i, j \in I$ such that $i \leq j$ and $b \in A_{j}$, the map $f_{i j}: A_{i} \rightarrow A_{j}$ defined by the rule $f_{i j}(x)=p(x, b)$ is a homomorphism. The definition of $f_{i j}$ is independent from the choice of $b$, since $p(a, b)=p(a, c)$, for all $a \in A_{i}$ and $c \in A_{j}$. A is isomorphic to the Płonka sum over the direct system

$$
\mathfrak{T}=\left\langle\left\{\mathbf{A}_{i}\right\}_{i \in I},\langle I, \leq\rangle,\left\{f_{i, j}: i, j \in I \text { and } i \leq j\right\}\right\rangle .
$$

Thus, we have:

Theorem 9 [22, Thm. III] Let $\mathcal{V}$ be a variety of type $\mathcal{L}$, let $\varphi(x, y)$ be an $\mathcal{L}$-formula in two variables, and let $\mathbf{A} \in \mathcal{V}$. Moreover, let $\mathcal{V}^{\prime}$ be the variety that is axiomatised relative to $\mathcal{V}$ by the equation $\varphi(x, y) \approx x$. Then $\varphi^{\mathbf{A}}$ is a partition function in $\mathbf{A}$ iff $\mathbf{A}$ is isomorphic to the Ptonka sum over a direct system of algebras from $\mathcal{V}^{\prime}$ (called the fibres of the Ptonka sum).

Płonka sums have an interesting connection with the theory of regular equations. Recall that an $\mathcal{L}$-equation $\varphi \approx \psi$ is said to be regular in case $\operatorname{Var}(\varphi)=\operatorname{Var}(\psi)$. 
Given a variety $\mathcal{V}$ of type $\mathcal{L}$, we denote by $R(\mathcal{V})$ the variety satisfying exactly the regular $\mathcal{L}$-equations satisfied by $\mathcal{V}$. This variety is called the regularisation of $\mathcal{V}$. It can be proved that an algebra is in $R(\mathcal{V})$ iff it can be represented as a Płonka sum over a direct system of algebras from $\mathcal{V}[24]$.

\subsection{Involutive Bisemilattices}

Distributive bisemilattices were introduced by Płonka under the name of distributive quasilattices in [21] as a substantive application of the techniques reviewed in the previous subsection. Distributive bisemilattices are the algebras that are representable as Płonka sums over direct systems of distributive lattices and include as limit cases both distributive lattices and semilattices. A generic distributive bisemilattice may fail non-regular identities in the language of lattices: a typical example is the absorption law $x \approx x \wedge(x \vee y)$. Involutive bisemilattices, introduced and investigated in [1], are the algebras that are representable as Płonka sums over direct systems of Boolean algebras - or, which is the same, the regularisations of Boolean algebras. They stand out as the algebraic counterpart of the paraconsistent three-valued logic PWK, the logic over the weak Kleene tables with two designated values.

Definition 10 An involutive bisemilattice is an algebra $\mathbf{A}=\langle A, \wedge, \vee, \neg, 0,1\rangle$ of type $\mathcal{L}_{0}$ satisfying the following equations:

(1) $x \vee x \approx x$

(2) $x \vee y \approx y \vee x$;

(3) $x \vee(y \vee z) \approx(x \vee y) \vee z$;

(4) $\neg \neg x \approx x$;

(5) $x \wedge y \approx \neg(\neg x \vee \neg y)$;

(6) $x \wedge(\neg x \vee y) \approx x \wedge y$;

(7) $0 \vee x \approx x$

(8) $1 \approx \neg 0$.

Thus, the class of involutive bisemilattices is a variety, which we denote by $\mathcal{I B S} \mathcal{L}$.

One can readily see that every involutive bisemilattice has, in particular, the structure of a join semilattice with zero, by virtue of axioms 1-3 and 7. More than that, the negation and constant-free reduct of an arbitrary involutive bisemilattice is a distributive bisemilattice (lattice distribution is provable from the axioms in Definition 10).

Two notable subvarieties of $\mathcal{I B S} \mathcal{L}$ (actually, as it happens to be, the unique two nontrivial subvarieties) are the variety $\mathcal{B} \mathcal{A}$ of Boolean algebras, axiomatised relative to $\mathcal{I B S} \mathcal{L}$ by one of the equivalent identities $x \wedge(x \vee y) \approx x$ or $x \vee \neg x \approx 1$, and the variety $\mathcal{S} \mathcal{L}$ of semilattices with zero, axiomatised relative to $\mathcal{I B S} \mathcal{L}$ by one of the equivalent identities $0 \approx 1, x \approx \neg x, x \wedge(x \vee y) \approx x \wedge y$ or $x \wedge y \approx x \vee y$. Also, in any involutive bisemilattice $\mathbf{A}$ the two relations defined, for all $a, b \in A$, by

$$
\begin{aligned}
& a \leq_{\vee} b \text { iff } a \vee b=b \text { and } \\
& a \leq_{\wedge} b \text { iff } a \wedge b=a
\end{aligned}
$$


are both semilattice orders on $A$. They coincide iff $\mathbf{A}$ is a Boolean algebra; they are dual to each other iff $\mathbf{A}$ is a semilattice with zero.

$\mathcal{I B S} \mathcal{L}$ is generated as a variety (but not as a quasivariety) by the 3-element algebra WK, with universe $\left\{0, \frac{1}{2}, 1\right\}$ and with the following tables:

\begin{tabular}{|c|c|c|c|c|c|c|c|c|c|}
\hline$\wedge$ & 0 & $1 / 2$ & 1 & V & 0 & $1 / 2$ & 1 & $\neg$ & 0 \\
\hline 0 & 0 & $1 / 2$ & 0 & 0 & 0 & $1 / 2$ & 1 & 0 & 1 \\
\hline $1 / 2$ & $1 / 2$ & $1 / 2$ & $1 / 2$ & $1 / 2$ & $1 / 2$ & $1 / 2$ & $1 / 2$ & $1 / 2$ & $1 / 2$ \\
\hline 1 & 0 & $1 / 2$ & 1 & 1 & 1 & $1 / 2$ & 1 & 1 & 0 \\
\hline
\end{tabular}

The relationships between the class of involutive bisemilattices and the paraconsistent non-protoalgebraic logic PWK $=\left\langle\mathbf{F m}\left(\mathcal{L}_{0}\right), \vdash\left\langle\mathbf{W K},\left\{1, \frac{1}{2}\right\}\right\rangle\right\rangle$ are clarified in [1]; its elucidation would require some concepts and tools from second-order AAL, which lie outside the scope of the present paper.

If $\mathbf{A}$ is any involutive bisemilattice, it is possible to check that the function $p(a, b)=a \wedge(a \vee b)$ is a partition function on A. According to Theorem 9 , thus, we get:

Theorem $11 \mathbf{A} \in \mathcal{I B S} \mathcal{L}$ iff $\mathbf{A}$ is isomorphic to the Płonka sum over a direct system of Boolean algebras.

Any involutive bisemilattice $\mathbf{A}$ falls under one of the following three cases, in terms of its Płonka sum representation:

(1) A has only one Boolean fibre. In this case, $\mathbf{A}$ is a Boolean algebra.

(2) All the Boolean fibres of $\mathbf{A}$ are trivial. In this case, $\mathbf{A}$ satisfies the equation $x \wedge y \approx x \vee y$, and hence it is a semilattice with zero.

(3) A has at least two nontrivial Boolean fibres. If so, WK is isomorphic to a subalgebra of a quotient of $\mathbf{A}$, whence the only subvariety of $\mathcal{I} \mathcal{B S} \mathcal{L}$ containing $\mathbf{A}$ is $\mathcal{I B S} \mathcal{L}$ itself.

We now introduce a construction on involutive bisemilattices. In analogy with other constructions in the broad family of twist products (see e.g. [4]), it yields an algebra in an expanded type, whose universe is the Cartesian product of the arguments, and whose operations are partly internal (meaning operations in the original type, defined componentwise) and partly external (operations not in the original type, involving an interplay between the components).

Definition 12 Let $\mathbf{A}, \mathbf{B} \in \mathcal{I B S} \mathcal{L}$. The I-product of $\mathbf{A}$ and $\mathbf{B}$ is the algebra $\mathbf{A} \odot \mathbf{B}=$ $\langle A \times B, \wedge, \vee, \rightarrow, \neg, 0,1\rangle$, of type $\mathcal{L}_{1}=\langle 2,2,2,1,0,0\rangle$, such that:

(1) its $\langle\wedge, \vee, \neg, 0,1\rangle$-reduct is the direct product $\mathbf{A} \times \mathbf{B}$;

(2) For all $a_{1}, a_{2} \in A$ and all $b_{1}, b_{2} \in B$,

$$
\left\langle a_{1}, b_{1}\right\rangle \rightarrow\left\langle a_{2}, b_{2}\right\rangle=\left\{\begin{array}{l}
\left\langle\neg^{\mathbf{A}} a_{1} \vee^{\mathbf{A}} a_{2}, \neg^{\mathbf{B}} b_{1} \vee^{\mathbf{B}} b_{2}\right\rangle, \text { if } b_{1} \leq_{\wedge}^{\mathbf{B}} b_{2} ; \\
\left\langle 0^{\mathbf{A}}, \neg^{\mathbf{B}} b_{1} \vee^{\mathbf{B}} b_{2}\right\rangle, \text { otherwise. }
\end{array}\right.
$$


Hereafter, for $\mathcal{K}, \mathcal{K}^{\prime} \subseteq \mathcal{I} \mathcal{B S} \mathcal{L}$, we denote by $\mathcal{K} \odot \mathcal{K}^{\prime}$ the class

$$
I\left(\left\{\mathbf{A} \odot \mathbf{B}: \mathbf{A} \in \mathcal{K}, \mathbf{B} \in \mathcal{K}^{\prime}\right\}\right) \text {. }
$$

\subsection{Demodalised Analytic Implication}

As we recalled in our introduction, the logic DAI of demodalised analytic implication is a containment logic that can be viewed as an extension of classical logic, rather than a subclassical logic. There are several equivalent ways to formally introduce DAI; what is perhaps the most perspicuous one is via the set-assignment semantics given by Richard Epstein [10], who independently rediscovered DAI under the heading of "dependence logic".

Hereafter, given a set $X$, we denote by $\mathbf{S}_{X}$ the join semilattice with zero (understood as an algebra in the type $\mathcal{L}_{0}$ ) of subsets of $X$, and by $S_{X}$ its universe. The next definition is essentially the one in [10] and in [9, p. 20].

Definition 13 A dependence model for $\mathcal{L}_{1}$ is a triple $\mathfrak{M}=\langle\mathfrak{S}, \mathfrak{v}, \mathfrak{s}\rangle$, where $\mathfrak{S}$ is a countable set and the maps $\mathfrak{v}: \operatorname{Var}\left(\mathcal{L}_{1}\right) \rightarrow\{0,1\}$ and $\mathfrak{s}: \operatorname{Var}\left(\mathcal{L}_{1}\right) \rightarrow S_{\mathfrak{S}}$ are extended to $F m\left(\mathcal{L}_{1}\right)$ as follows:

- $\mathfrak{s}(\varphi)=\bigcup\{\mathfrak{s}(x): x \in \operatorname{var}(\varphi)\}$

- $\mathfrak{v}(0)=0$ and $\mathfrak{v}(1)=1$;

- $\mathfrak{v}(\neg \varphi)=1$ if and only if $\mathfrak{v}(\varphi)=0$;

- $\mathfrak{v}(\varphi \wedge \psi)=1$ if and only if $\mathfrak{v}(\varphi)=\mathfrak{v}(\psi)=1$;

- $\mathfrak{v}(\varphi \vee \psi)=1$ if and only if either $\mathfrak{v}(\varphi)=1$ or $\mathfrak{v}(\psi)=1$;

- $\mathfrak{v}(\varphi \rightarrow \psi)=1$ if and only if $\mathfrak{s}(\psi) \subseteq \mathfrak{s}(\varphi)$ and (not both $\mathfrak{v}(\varphi)=1$ and $\mathfrak{v}(\psi)=$ $0)$.

Definition 14 DAI is the logic $\left\langle\mathbf{F m}\left(\mathcal{L}_{1}\right), \vdash_{\text {DAI }}\right\rangle$, where for all $\Gamma \cup\{\varphi\} \subseteq F m\left(\mathcal{L}_{1}\right)$, $\Gamma \vdash_{\text {DAI }} \varphi$ iff for all dependence models $\mathfrak{M}=\langle\mathfrak{S}, \mathfrak{v}, \mathfrak{s}\rangle$, if $\mathfrak{v}(\psi)=1$ for all $\psi \in \Gamma$, then $\mathfrak{v}(\varphi)=1$.

It is immediate from the definitions that whenever $\Gamma \cup\{\varphi\} \subseteq F m\left(\mathcal{L}_{0}\right)$, we have that $\Gamma \vdash_{\text {DAI }} \varphi$ iff $\Gamma \vdash_{\mathrm{CL}} \varphi$.

\section{Algebraic Analysis of DAI}

\subsection{Implicative Involutive Bisemilattices}

In this section we consider an expansion of involutive bisemilattices by an additional binary operation of implication, meant to algebraically represent demodalised analytic implication. Although, strictly speaking, such implicative involutive bisemilattices do not lend themselves to an analysis in terms of Płonka sums, it turns out that a suitable variation on this theme can provide us with a handy semantics that will be conveniently exploited below. 
Definition 15 An implicative involutive bisemilattice is an algebra

$$
\mathbf{A}=\langle A, \wedge, \vee, \rightarrow, \neg, 0,1\rangle
$$

of type $\mathcal{L}_{1}$ that satisfies the following conditions:

(1) The reduct $\mathbf{A}^{-}=\langle A, \wedge, \vee, \neg, 0,1\rangle$ is an involutive bisemilattice.

(2) A satisfies the following quasiequations:

$$
\begin{aligned}
& \text { (I1) } x \approx \neg x \Rightarrow y \approx z ; \\
& \text { (I2) } x \wedge(x \vee y) \approx x \Rightarrow x \rightarrow y \approx \neg x \vee y ; \\
& \text { (I3) } x \rightarrow y \approx(x \rightarrow y) \vee 1 \Rightarrow x \wedge(x \vee y) \approx x .
\end{aligned}
$$

The quasivariety of implicative involutive bisemilattices will be denoted by $\mathcal{I} \mathcal{B S} \mathcal{L}$.

Observe that, whenever $\mathbf{A} \in \mathcal{I} \mathcal{I} \mathcal{B S} \mathcal{L}$, the arrow-free reduct $\mathbf{A}^{-}$of $\mathbf{A}$ is an involutive bisemilattice whose Płonka sum representation either contains no trivial Boolean fibres, or, by (I1), is the trivial algebra. In particular, neither WK (Section 2.3) nor any nontrivial semilattice with zero is the $\mathcal{L}_{0}$-reduct of any member of $\mathcal{I} \mathcal{I} \mathcal{S} \mathcal{L}$.

Example 16 We leave it to the reader to check that every member of

$$
\left(\mathcal{B A} \backslash\left\{\mathbf{B}_{1}\right\}\right) \odot \mathcal{S L}
$$

(see Definition 12) is an implicative involutive bisemilattice.

We now want to use a variant of the Płonka sum construction to obtain implicative involutive bisemilattices. As a preliminary step, following the lead of [2], we adjust the notions of a direct system and of a Płonka sum to the case in which the summands are not algebras but logical matrices-in particular, matrix models of classical logic.

Definition 17 Let $\left\{\left\langle\mathbf{A}_{i}, F_{i}\right\rangle\right\}_{i \in I} \subseteq \operatorname{Mod}(\mathrm{CL})$. The triple

$$
\mathfrak{T}^{m}=\left\langle\left\{\left\langle\mathbf{A}_{i}, F_{i}\right\rangle\right\}_{i \in I},\langle I, \leq, \perp\rangle,\left\{f_{i, j}: i, j \in I \text { and } i \leq j\right\}\right\rangle
$$

is a $d$-direct system of matrices of type $\mathcal{L}_{0}$ in case:

(1) $\langle I, \leq, \perp\rangle$ is a join semilattice with zero;

(2) If $i, j \in I$ and $i \neq j$, then $\mathbf{A}_{i} \cap \mathbf{A}_{j}=\emptyset$;

(3) Each $f_{i, j}$ is a homomorphism from $\mathbf{A}_{i}$ to $\mathbf{A}_{j}$ such that

$$
f_{i, i}(x)=x \text { and } f_{j, k}\left(f_{i, j}(x)\right)=f_{i, k}(x) ;
$$

moreover, for all $i, j \in I, f_{i, j}^{-1}\left(F_{j}\right)=F_{i}$.

Definition 18 The Ptonka sum over a d-direct system of matrices of type $\mathcal{L}_{0}$

$$
\mathfrak{T}^{m}=\left\langle\left\{\left\langle\mathbf{A}_{i}, F_{i}\right\rangle\right\}_{i \in I},\langle I, \leq, \perp\rangle,\left\{f_{i, j}: i, j \in I \text { and } i \leq j\right\}\right\rangle
$$


is the matrix, also of type $\mathcal{L}_{0}, \mathbf{P}\left(\mathfrak{T}^{m}\right)=\left\langle\mathbf{P}(\mathfrak{T}), \bigcup_{i \in I} F_{i}\right\rangle$, where $\mathbf{P}(\mathfrak{T})$ is the Płonka sum over

$$
\mathfrak{T}=\left\langle\left\{\mathbf{A}_{i}\right\}_{i \in I},\langle I, \leq, \perp\rangle,\left\{f_{i, j}: i, j \in I \text { and } i \leq j\right\}\right\rangle
$$

Throughout the rest of the paper, given $\mathbf{A} \cong \mathbf{P}(\mathfrak{T}), h: \mathbf{F m}\left(\mathcal{L}_{0}\right) \rightarrow \mathbf{A}$ and $\varphi \in$ $F m\left(\mathcal{L}_{0}\right)$, we denote by $i_{h}(\varphi)$ the index of the fibre that contains $h(\varphi)$.

Lemma 19 Let $\mathbf{P}\left(\mathfrak{T}^{m}\right)=\left\langle\mathbf{P}(\mathfrak{T}), \bigcup_{i \in I} F_{i}\right\rangle$ be a Ptonka sum over a d-direct system $\mathfrak{T}^{m}$ of matrices. Then $\mathbf{P}\left(\mathfrak{T}^{m}\right) \in \operatorname{Mod}(\mathrm{CL})$.

Proof Consider $\mathbf{P}\left(\mathfrak{T}^{m}\right)=\left\langle\mathbf{P}(\mathfrak{T}), \bigcup_{i \in I} F_{i}\right\rangle$ as in the statement of the lemma and let $\Gamma \vdash_{\mathrm{CL}} \varphi$. Let also $h: \mathbf{F m}\left(\mathcal{L}_{0}\right) \rightarrow \mathbf{A}$ be a homomorphism such that $h[\Gamma] \subseteq \bigcup_{i \in I} F_{i}$.

Suppose towards a contradiction that $h(\varphi) \notin \bigcup_{i \in I} F_{i}$. As CL is finitary, without any loss of generality we can take $\Gamma$ to be the finite set $\left\{\gamma_{1}, \ldots, \gamma_{n}\right\}$. We compute $k=$ $i_{h}\left(\gamma_{1}\right) \vee \ldots \vee i_{h}\left(\gamma_{n}\right)$ and let $i=i_{h}(\varphi)$. Observe that, as $\mathfrak{T}^{m}$ is a d-direct system, the fact that

$$
h\left(\gamma_{1}\right) \in F_{i_{h}\left(\gamma_{1}\right)}, \ldots, h\left(\gamma_{n}\right) \in F_{i_{h}\left(\gamma_{n}\right)}
$$

implies

$$
f_{i_{h}\left(\gamma_{1}\right) k}\left(h\left(\gamma_{1}\right)\right) \in F_{k}, \ldots, f_{i_{h}\left(\gamma_{n}\right) k}\left(h\left(\gamma_{n}\right)\right) \in F_{k} .
$$

Now, fixing $j=i \vee k$, clearly we have $f_{k j}(h[\Gamma]) \subseteq F_{j}$. Moreover, as $h(\varphi) \notin F_{i}$, $f_{i j}(h(\varphi)) \in A_{j} \backslash F_{j}$. Define now a homomorphism $v: \mathbf{F m}\left(\mathcal{L}_{0}\right) \rightarrow \mathbf{A}_{i}$ as

$$
v(x)=\left\{\begin{array}{l}
f_{i_{h}(x) j} \circ h(x), \text { if } x \in \operatorname{Var}(\Gamma \cup\{\varphi\}), \\
a \in A_{j}, \text { otherwise. }
\end{array}\right.
$$

Then clearly $v[\Gamma] \subseteq F_{j}$, while $v(\varphi) \in A_{j} \backslash F_{j}$, against the fact that $\Gamma \vdash_{\mathrm{CL}} \varphi$ and $\left\langle\mathbf{A}_{j}, F_{j}\right\rangle \in \operatorname{Mod}(\mathrm{CL})$. This is a contradiction, so $h(\varphi) \in F$.

Now, let

$$
\mathfrak{T}^{m}=\left\langle\left\{\left\langle\mathbf{A}_{i}, F_{i}\right\rangle\right\}_{i \in I},\langle I, \leq, \perp\rangle,\left\{f_{i, j}: i, j \in I \text { and } i \leq j\right\}\right\rangle
$$

be a d-direct system of matrices.

Lemma 20 If $\mathbf{P}\left(\mathfrak{T}^{m}\right)$ is non-trivial, then so is $\left\langle\mathbf{A}_{i}, F_{i}\right\rangle$ for each $i \in I$.

Proof We reason by contraposition. So, suppose there is a trivial fibre $\left\langle\mathbf{A}_{i}, F_{i}\right\rangle$. We show that an arbitrary fibre $\left\langle\mathbf{A}_{k}, F_{k}\right\rangle$ is trivial as well, i.e. $\langle\mathbf{A}, F\rangle$ is trivial. Fix $j=$ $i \vee k$. Since $A_{i}=F_{i}$ for any $v: \mathbf{F m}\left(\mathcal{L}_{0}\right) \rightarrow \mathbf{A}_{i}$ we have that $v(x \wedge \neg x) \in F_{i}$ and so $f_{i j}(v(x \wedge \neg x)) \in F_{j}$. The fact that $x \wedge \neg x \vdash_{\text {CL }} y$ for every $y \in \operatorname{Var}\left(\mathcal{L}_{1}\right)$, together with $\left\langle\mathbf{A}_{j}, F_{j}\right\rangle \in \operatorname{Mod}(\mathrm{CL})$, entails that $A_{j}=F_{j}$. Indeed, if there were $c \in A_{j} \backslash F_{j}$, 
we would define $h: \mathbf{F m}\left(\mathcal{L}_{0}\right) \rightarrow \mathbf{A}_{j}$ such that $h(x)=f_{i j} \circ v(x)$ and $h(y)=c$, whence $h(x \wedge \neg x) \in F_{j}, h(y) \notin F_{j}$. This proves that if $A_{i}=F_{i}$ then $A_{j}=F_{j}$ for each $i \leq j$. Moreover, as by Definition $13 f_{k j}^{-1}\left[F_{j}\right]=F_{k}$ we obtain $A_{k}=F_{k}$. This proves that each fibre is trivial.

In view of Theorem 11, the algebra reducts of Płonka sums over d-direct systems of matrices are involutive bisemilattices, and as such they do not contain any primitive implication connective. In particular, they do not belong to $\mathcal{I} \mathcal{B} \mathcal{S} \mathcal{L}$. We now show how to obtain certain implicative involutive bisemilattices out of such algebra reducts.

Definition 21 A $P$-implicative involutive bisemilattice is an algebra $\mathbf{A}_{\mathbf{P}\left(\mathfrak{T}^{m}\right)}$ of type $\mathcal{L}_{1}$ such that:

(1) its $\mathcal{L}_{0}$-reduct is isomorphic to the algebra reduct of the Plonka sum $\mathbf{P}\left(\mathfrak{T}^{m}\right)$ over a d-direct system of matrices

$$
\mathfrak{T}^{m}=\left\langle\left\{\left\langle\mathbf{A}_{i}, F_{i}\right\rangle\right\}_{i \in I},\langle I, \leq, \perp\rangle,\left\{f_{i, j}: i, j \in I \text { and } i \leq j\right\}\right\rangle
$$

such that either $I=\{1\}$ or for no $i \in I, \mathbf{A}_{i}=\mathbf{B}_{1}$;

(2) the binary operation $\rightarrow \mathbf{A}^{\left.\mathbf{A}_{\left(\mathfrak{T}^{m}\right.}\right)}$ is defined for all $a, b \in \bigcup\left\{A_{i}\right\}_{i \in I}$ by the stipulation

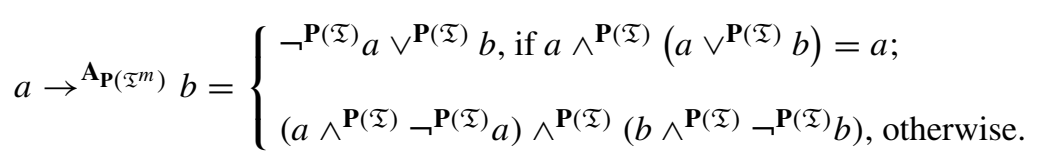

The class of P-implicative involutive bisemilattices will be denoted by $\mathcal{P} \mathcal{I} \mathcal{B S}$. Next, we prove that this denomination is not a misnomer.

Theorem 22 Every P-implicative involutive bisemilattice $\mathbf{A}_{\mathbf{P}\left(\mathfrak{T}^{m}\right)}$ belongs to $\mathcal{I} \mathcal{I} \mathcal{S} \mathcal{L}$.

Proof Condition (1) in Definition 15 is a direct consequence of Theorem 11 and Definition 15 itself. We now verify the quasiequations in Condition (2).

As regards (I1), suppose that $a \in A \mathbf{P}\left(\mathfrak{T}^{m}\right)$ is such that $a=\neg a$. Then the fibre containing $a$ is a trivial Boolean algebra and thus $\mathbf{A}_{\mathbf{P}\left(\mathfrak{T}^{m}\right)}$ is also trivial, by Definition 21. For (I2), let $a, b \in A_{\mathbf{P}\left(\mathfrak{T}^{m}\right)}$ be such that $a \wedge(a \vee b)=a$. Then by Definition 21, $a \rightarrow b=\neg a \vee b$. Finally, for (I3), let $a, b \in A_{\mathbf{P}\left(\mathfrak{T}^{m}\right)}$ be such that $a \rightarrow b=(a \rightarrow b) \vee$ 1. If $\mathbf{A}_{\mathbf{P}\left(\mathfrak{T}^{m}\right)}$ is trivial, the quasiequation is satisfied. If it is not, let $a \in A_{i}, b \in A_{j}$. This means that $a \rightarrow b$ is the top element of the fibre $\mathbf{A}_{k}$, where $k=i \vee j$. Were it the case that $a \wedge(a \vee b) \neq a$, by Definition 21 the fibre $\mathbf{A}_{k}$ would be trivial and then $\mathbf{A}_{\mathbf{P}\left(\mathfrak{T}^{m}\right)}$ would also be trivial, a contradiction.

We postpone further investigation of these structures, and of their relationships with DAI, until after we have examined some alternative semantics for this logic.

\subsection{Some Completeness Theorems}

The aim of this section is to provide two algebraic semantics for DAI. The former is based on P-implicative involutive bisemilattices, while the latter uses the product construction in Section 2.3. The completeness of these semantics will be proved 
by showing their equivalence to the more customary modellings of DAI, either in terms of dependence models or in terms of the models discussed by Dunn in $[8, \S 6]$ (although this is not the main approach employed in that paper). It will be observed that such models are a special case of models based on products of involutive bisemilattices.

We first need a technical lemma.

Lemma 23 (1) Let $\mathfrak{M}=\langle\mathfrak{S}, \mathfrak{v}, \mathfrak{s}\rangle$ be a dependence model for $\mathcal{L}_{1}$. Then there exists a countable set $X$ such that the map $v^{*}$, defined by

$$
v^{*}(x)=\langle\mathfrak{v}(x), \mathfrak{s}(x)\rangle,
$$

belongs to Hom $\left(\mathbf{F m}\left(\mathcal{L}_{1}\right), \mathbf{B}_{2} \odot \mathbf{S}_{X}\right)$.

(2) If $X$ is a countable set and $v \in \operatorname{Hom}\left(\mathbf{F m}\left(\mathcal{L}_{1}\right), \mathbf{B}_{2} \odot \mathbf{S}_{X}\right)$, then $g(M)=$ $\left\langle X, \pi_{1} \circ v, \pi_{2} \circ v\right\rangle$, where $\pi_{1}$ (resp. $\pi_{2}$ ) denote the operation of left (resp. right) projection, is a dependence model for $\mathcal{L}_{1}$.

Proof (1). We only check that $v^{*}$ respects conjunction and implication.

$$
\begin{aligned}
& v^{*}(\varphi \wedge \psi)=\langle\mathfrak{v}(\varphi \wedge \psi), \mathfrak{s}(\varphi \wedge \psi)\rangle \\
& =\left\langle\mathfrak{v}(\varphi) \wedge^{\mathbf{B}_{2}} \mathfrak{v}(\psi), \mathfrak{s}(\varphi) \vee^{\mathbf{S}_{\mathfrak{S}}} \mathfrak{s}(\psi)\right\rangle \\
& =\langle\mathfrak{v}(\varphi), \mathfrak{s}(\varphi)\rangle \wedge^{\mathbf{B}_{2} \odot \mathbf{S}_{\mathfrak{S}}}\langle\mathfrak{v}(\psi), \mathfrak{s}(\psi)\rangle \\
& =v^{*}(\varphi) \wedge^{\mathbf{B}_{2} \odot \mathbf{S}_{\mathfrak{S}}} v^{*}(\psi) \text {; }
\end{aligned}
$$

moreover, if $\mathfrak{s}(\psi) \subseteq \mathfrak{s}(\varphi)$ and $\mathfrak{v}(\neg \varphi \vee \psi)=\neg^{\mathbf{B}_{2}} \mathfrak{v}(\varphi) \vee^{\mathbf{B}_{2}} \mathfrak{v}(\psi)=1$, then

$$
\begin{aligned}
v^{*}(\varphi \rightarrow \psi) & =\langle\mathfrak{v}(\varphi \rightarrow \psi), \mathfrak{s}(\varphi \rightarrow \psi)\rangle=\left\langle 1^{\mathbf{B}_{2}}, \mathfrak{s}(\varphi) \vee^{\mathbf{S}_{\mathfrak{S}}} \mathfrak{s}(\psi)\right\rangle \\
& =\langle\mathfrak{v}(\varphi), \mathfrak{s}(\varphi)\rangle \rightarrow{ }^{\mathbf{B}_{2} \odot \mathbf{S}_{\mathfrak{S}}}\langle\mathfrak{v}(\psi), \mathfrak{s}(\psi)\rangle=v^{*}(\varphi) \rightarrow{ }^{\mathbf{B}_{2} \odot \mathbf{S}_{\mathfrak{S}}} v^{*}(\psi),
\end{aligned}
$$

while otherwise we have that $v^{*}(\varphi \rightarrow \psi)=\left\langle 0^{\mathbf{B}_{2}}, \mathfrak{s}(\varphi) \vee^{\mathbf{S}_{\mathfrak{S}}} \mathfrak{s}(\psi)\right\rangle=$ $v^{*}(\varphi) \rightarrow \mathbf{B}_{2} \odot \mathbf{S}_{\mathfrak{S}} v^{*}(\psi)$.

(2). Again, it suffices to show that $\pi_{1} \circ v$ and $\pi_{2} \circ v$ obey the clauses in Definition 13. $\pi_{2} \circ v: F m\left(\mathcal{L}_{1}\right) \rightarrow \wp(X)$ is such that

$$
\pi_{2} \circ v(\varphi)=\bigcup\left\{\pi_{2} \circ v(p): p \in \operatorname{Var}(\varphi)\right\} .
$$

As regards $\pi_{1} \circ v$, we confine ourselves to showing that $\pi_{1} \circ v(\varphi \rightarrow \psi)=1$ iff $\pi_{1} \circ v(\neg \varphi \vee \psi)=1$ and $\pi_{2} \circ v(\psi) \subseteq \pi_{2} \circ v(\varphi)$. However, if the right-hand side of the biconditional is true,

$$
\begin{aligned}
v(\varphi \rightarrow \psi) & =v(\varphi) \rightarrow{ }^{\mathbf{B}_{2} \odot \mathbf{S}_{X}} v(\psi) \\
& =\left\langle\pi_{1} \circ v(\varphi), \pi_{2} \circ v(\varphi)\right\rangle \rightarrow^{\mathbf{B}_{2} \odot \mathbf{S}_{X}}\left\langle\pi_{1} \circ v(\psi), \pi_{2} \circ v(\psi)\right\rangle \\
& =\left\langle\neg^{\mathbf{B}_{2}}\left(\pi_{1} \circ v(\varphi)\right) \vee^{\mathbf{B}_{2}} \pi_{1} \circ v(\psi), \pi_{2} \circ v(\varphi) \vee \pi_{2} \circ v(\psi)\right\rangle \\
& =\left\langle 1^{\mathbf{B}_{2}}, \pi_{2} \circ v(\varphi) \vee \pi_{2} \circ v(\psi)\right\rangle,
\end{aligned}
$$

so $\pi_{1} \circ v(\varphi \rightarrow \psi)=1$. Similarly, it is easy to check that if the right-hand side of the biconditional is false, then $\pi_{1} \circ v(\varphi \rightarrow \psi)=0$. 
We now semantically introduce two logics that we want to prove coincident with DAI. With an eye to doing so, we provide a recipe for associating logics to classes of implicative involutive bisemilattices.

Definition 24 If $\mathcal{K} \subseteq \mathcal{I I B S} \mathcal{L}$, let $\mathrm{DAI}_{\mathcal{K}}$ be the logic $\left\langle\mathbf{F m}\left(\mathcal{L}_{1}\right), \vdash_{\mathrm{DAI}_{\mathcal{K}}}\right\rangle$, where, for all $\Gamma \cup\{\varphi\} \subseteq F m\left(\mathcal{L}_{1}\right)$,

$$
\begin{aligned}
\Gamma \vdash_{\mathrm{DAI}_{\mathcal{K}}} \varphi \text { iff } & \text { for every } \mathbf{A} \in \mathcal{K} \text { and every } h \in \operatorname{Hom}\left(\mathbf{F m}\left(\mathcal{L}_{1}\right), \mathbf{A}\right), \\
& \text { if } 1^{\mathbf{A}} \leq_{\vee}^{\mathbf{A}} h(\gamma) \text { for all } \gamma \in \Gamma, \text { then } 1^{\mathbf{A}} \leq_{\vee}^{\mathbf{A}} h(\varphi) .
\end{aligned}
$$

Let us note that the subclasses of $\mathcal{I} \mathcal{I B S} \mathcal{L}$ of immediate concern, for us, are $\mathcal{P r}=\left(\mathcal{B} \mathcal{A} \backslash\left\{\mathbf{B}_{1}\right\}\right) \odot \mathcal{S} \mathcal{L}$ and $\mathcal{P} r^{x}=\left\{\mathbf{B}_{2} \odot \mathbf{S}_{X}: X\right.$ a countable set $\}$. The corresponding logics $\mathrm{DAI}_{\mathcal{P} r}$ and $\mathrm{DAI}_{\mathcal{P} r^{x}}$ correspond to the logics respectively determined by certain I-products of Section 2.3 and by the Dunn models referred to in the introduction to this subsection. Observe that $\mathrm{DAI}_{\mathcal{P} r}$ can also be viewed as the logic determined by the class of all matrices of the form $\langle\mathbf{B} \odot \mathbf{S}, F\rangle$, where $\mathbf{B}$ is a nontrivial Boolean algebra, $\mathbf{S}$ is a semilattice with zero, and $F=$ $\left\{\langle a, b\rangle \in B \times S: 1^{\mathbf{B}}=a\right.$ and $\left.1^{\mathbf{S}}=0^{\mathbf{S}} \leq^{\mathbf{S}} b\right\}$. Since the latter condition is always satisfied, such DAI $\mathcal{P}_{\mathcal{P} r}$-filters have the form $\left\{\langle a, b\rangle \in B \times S: 1^{\mathbf{B}}=a\right\}$. Analogously, $\mathrm{DAI}_{\mathcal{P r}^{x}}$ is the logic determined by the class of all matrices of the form $\left\langle\mathbf{B}_{2} \odot \mathbf{S}_{X}, F\right\rangle$, where $F=\left\{\langle a, b\rangle \in B_{2} \times S_{X}: 1^{\mathbf{B}_{2}}=a\right\}$.

Theorem 25 The logics $\mathrm{DAI}, \mathrm{DAI}_{\mathcal{P} r}$ and $\mathrm{DAI}_{\mathcal{P}} x$ coincide.

Proof We first show the coincidence of DAI and DAI $\mathcal{P r}^{x}$. Suppose that $\Gamma \vdash_{\text {DAI }}$ $\varphi$. Then, according to Definition 14 , for every dependence model $\mathfrak{M}=\langle\mathfrak{S}, \mathfrak{v}, \mathfrak{s}\rangle$, if $\mathfrak{v}(\gamma)=1$ for all $\gamma \in \Gamma$, then $\mathfrak{v}(\varphi)=1$. With an eye to establishing that $\Gamma \vdash_{\operatorname{DAI}_{\mathcal{P}_{r} x}}$ $\varphi$, consider an arbitrary countable set $X$ and a given $v \in \operatorname{Hom}\left(\mathbf{F m}\left(\mathcal{L}_{1}\right), \mathbf{B}_{2} \odot \mathbf{S}_{X}\right)$ such that $1^{\mathbf{B}_{2} \odot \mathbf{S}_{X}} \leq_{\vee}^{\mathbf{B}_{2} \odot \mathbf{S}_{X}} v(\gamma)$, for all $\gamma \in \Gamma$. By our previous remarks, this holds iff $1^{\mathbf{B}_{2}}=\pi_{1} \circ v(\gamma)$, for all $\gamma \in \Gamma$. By Lemma 23.(2), $\left\langle X, \pi_{1} \circ v, \pi_{2} \circ v\right\rangle$ is a dependence model, and since $1^{\mathbf{B}_{2}}=\pi_{1} \circ v(\gamma)$ for all $\gamma \in \Gamma$, our assumption implies that $1^{\mathbf{B}_{2}}=$ $\pi_{1} \circ v(\varphi)$. This ensures that $1^{\mathbf{B}_{2} \odot \mathbf{S}_{X}} \leq_{\vee}^{\mathbf{B}_{2} \odot \mathbf{S}_{X}} v(\varphi)$.

Conversely, suppose that $\Gamma \nvdash_{\text {DAI }} \varphi$. Thus, there exists a dependence model $\mathfrak{M}=\langle\mathfrak{S}, \mathfrak{v}, \mathfrak{s}\rangle$ such that $\mathfrak{v}(\gamma)=1$ for all $\gamma \in \Gamma$, yet $\mathfrak{v}(\varphi)=0$. By Lemma 23.(1), $v^{*}(p)=\langle\mathfrak{v}(p), \mathfrak{s}(p)\rangle$ belongs to $\operatorname{Hom}\left(\mathbf{F m}\left(\mathcal{L}_{1}\right), \mathbf{B}_{2} \odot \mathbf{S}_{\mathfrak{S}}\right)$. Since $1^{\mathbf{B}_{2}}=\mathfrak{v}(\gamma)=$ $\pi_{1} \circ v^{*}(\gamma)$ for all $\gamma \in \Gamma$, then $1^{\mathbf{B}_{2} \odot \mathbf{S}_{\mathfrak{S}}} \leq_{\vee} \mathbf{B}_{2} \odot \mathbf{S}_{\mathfrak{S}} v^{*}(\gamma)$, for all $\gamma \in \Gamma$. However, it is not the case that $1^{\mathbf{B}_{2} \odot \mathbf{S}_{\mathfrak{S}}} \leq \leq_{V}^{\mathbf{B}_{2} \odot \mathbf{S}_{\mathfrak{S}}} v^{*}(\varphi)$. Therefore $\Gamma \nvdash_{\mathrm{DAI}_{\mathcal{P} r} x} \varphi$.

Since it is trivially the case that $\vdash_{\mathrm{DAI}_{\mathcal{P}_{r}}} \subseteq \vdash_{\mathrm{DAI}_{\mathcal{P}^{x}}}$, we prove the converse inclusion. Assume that $\Gamma \vdash_{\mathrm{DAI}_{\mathcal{P}_{r}} x} \varphi$; since DAI $=\mathrm{DAI}_{\mathcal{P}^{x}}, \Gamma$ can be assumed to be a finite set $\left\{\gamma_{1}, \ldots, \gamma_{n}\right\}$. Take a nontrivial Boolean algebra $\mathbf{B}$ and a semilattice with zero $\mathbf{S}$, together with $v \in \operatorname{Hom}\left(\mathbf{F m}\left(\mathcal{L}_{1}\right), \mathbf{B} \odot \mathbf{S}\right)$, such that $1^{\mathbf{B} \odot \mathbf{S}} \leq_{\vee}^{\mathbf{B} \odot \mathbf{S}} v(\gamma)$, for all $\gamma \in \Gamma$, but it is not the case that $1^{\mathbf{B} \odot \mathbf{S}} \leq_{\mathrm{V}}^{\mathbf{B} \odot \mathbf{S}} v(\varphi)$. By the structure of I-products, this means that either $1^{\mathbf{B}} \neq \pi_{1} \circ v(\varphi)$ or $1^{\mathbf{S}} \not^{\mathbf{S}} \pi_{2} \circ v(\varphi)$. If the former, then $\mathbf{B}_{2}$ falsifies the quasiequation $\gamma_{1} \approx 1 \& \ldots \& \gamma_{n} \approx 1 \Rightarrow \varphi \approx 1$, since $\mathbf{B}_{2}$ generates $\mathcal{B A}$ as a quasivariety. Call $v_{1}$ the falsifying valuation; then for any countable set $X$ and for any 
$v^{+} \in \operatorname{Hom}\left(\mathbf{F m}\left(\mathcal{L}_{1}\right), \mathbf{B}_{2} \odot \mathbf{S}_{X}\right)$ such that $\pi_{1} \circ v^{+}=v_{1}$, the algebra $\mathbf{B}_{2} \odot \mathbf{S}_{X} \in \mathcal{P} r^{x}$ will be such that $1^{\mathbf{B}_{2} \odot \mathbf{S}_{X}} \leq_{\mathrm{V}}^{\mathbf{B}_{2} \odot \mathbf{S}_{X}} v^{+}(\gamma)$ for all $\gamma \in \Gamma$, but $1^{\mathbf{B}_{2} \odot \mathbf{S}_{X}} \not_{\mathrm{V}} \mathbf{B}_{2} \odot \mathbf{S}_{X} v^{+}(\varphi)$, contradicting the assumption that $\Gamma \vdash \operatorname{DAI}_{\mathcal{P}_{r} x} \varphi$. If the latter, then some countable power $\mathbf{S}_{2}^{X}$ of the 2-element semilattice with zero $\mathbf{S}_{2}$ falsifies the same quasiequation, because $\mathbf{S}_{2}$ generates $\mathcal{S} \mathcal{L}$ as a quasivariety. Calling $v_{2}$ the falsifying valuation, we get another family of algebras of the form $\mathbf{B}_{2} \odot \mathbf{S}_{X}$, and of homomorphisms $v^{+*}$, where $\pi_{2} \circ v^{+*}=v_{2}$, that contradict once more the assumption $\Gamma \vdash_{\mathrm{DAI}_{\mathcal{P}_{r} x}} \varphi$.

Theorem 25 can be viewed as showing that each of the classes $\mathcal{P r}$ and $\mathcal{P} r^{x}$ is an algebraic semantics for DAI, under the set of defining equations $\tau(x)=$ $\{1 \vee x \approx x\}$. Our next step is proving that the class $\mathcal{P} \mathcal{I B S} \mathcal{L}$ of P-implicative involutive bisemilattices is an algebraic semantics for DAI as well.

\section{Theorem 26 The logics $\mathrm{DAI}$ and $\mathrm{DAI}_{\mathcal{P} \mathcal{I B S} \mathcal{L}}$ coincide.}

Proof Suppose that $\Gamma \vdash_{\text {DAI }} \varphi$; as above, w.l.o.g. $\Gamma$ can be taken to be finite. We resort once more to Definition 14, which guarantees that for every dependence model $\mathfrak{M}=\langle\mathfrak{S}, \mathfrak{v}, \mathfrak{s}\rangle$, if $\mathfrak{v}(\gamma)=1$ for all $\gamma \in \Gamma$, then $\mathfrak{v}(\varphi)=1$. Let now $\mathbf{A}=\mathbf{A}_{\mathbf{P}\left(\mathfrak{T}^{m}\right)} \in$ $\mathcal{P} \mathcal{I B S} \mathcal{L}$, where

$$
\mathfrak{T}^{m}=\left\langle\left\{\left\langle\mathbf{A}_{i}, F_{i}\right\rangle\right\}_{i \in I},\langle I, \leq, \perp\rangle,\left\{f_{i, j}: i, j \in I \text { and } i \leq j\right\}\right\rangle .
$$

Then notice that it also holds $\mathbf{A}=\mathbf{A}_{\mathbf{P}\left(\mathfrak{T}^{m^{\prime}}\right)}$

$$
\mathfrak{T}^{m^{\prime}}=\left\langle\left\{\left\langle\mathbf{A}_{i},\left\{1^{\mathbf{A}_{i}}\right\}\right\rangle\right\}_{i \in I},\langle I, \leq, \perp\rangle,\left\{f_{i, j}: i, j \in I \text { and } i \leq j\right\}\right\rangle
$$

and let $h \in \operatorname{Hom}\left(\mathbf{F m}\left(\mathcal{L}_{1}\right), \mathbf{A}\right)$ be such that $1^{\mathbf{A}} \leq_{\mathrm{V}}^{\mathbf{A}} h(\gamma)$ for all $\gamma \in \Gamma$. Since we are only interested in such members of $\mathcal{P} \mathcal{I B S} \mathcal{L}$ as can potentially falsify entailments with finitely many premisses, $\mathbf{A}$ can be taken to be countable. For $a \in A$, the notation $\downarrow a$ will be shorthand for

$$
\left\{b \in A: b \leq_{\vee}^{\mathbf{A}} a\right\} .
$$

Consider the triple $\mathfrak{M}_{\mathbf{A}}=\left\langle\mathfrak{S}_{\mathbf{A}}, \mathfrak{v}_{\mathbf{A}}, \mathfrak{s}_{\mathbf{A}}\right\rangle$, where:

$$
\begin{aligned}
& \mathfrak{S}_{\mathbf{A}}=\{\downarrow a: a \in A\} ; \\
& \mathfrak{v}_{\mathbf{A}}(x)=\left\{\begin{array}{l}
1 \text { if } h(x) \in \bigcup_{i \in I}\left\{1^{\mathbf{A}_{i}}\right\}, \\
0, \text { otherwise; }
\end{array}\right. \\
& \mathfrak{s}_{\mathbf{A}}(x)=\downarrow h(x) .
\end{aligned}
$$

We prove that $\mathfrak{M}_{\mathbf{A}}$ is a dependence model. The only nontrivial item to check is that for all formulas $\chi, \psi, \mathfrak{v}_{\mathbf{A}}(\chi \rightarrow \psi)=1$ if and only if $\mathfrak{s}_{\mathbf{A}}(\psi) \subseteq \mathfrak{s}_{\mathbf{A}}(\chi)$ and (not both $\mathfrak{v}_{\mathbf{A}}(\chi)=1$ and $\left.\mathfrak{v}_{\mathbf{A}}(\psi)=0\right)$.

We claim that for all formulas $\chi, \psi, i_{h}(\chi) \leq i_{h}(\psi)$ if and only if $\mathfrak{s}_{\mathbf{A}}(\chi) \subseteq$ $\mathfrak{s}_{\mathbf{A}}(\psi)$. From left to right, suppose $i_{h}(\chi) \leq i_{h}(\psi)$ and let $\operatorname{Var}(\chi)=\left\{x_{1}, \ldots, x_{n}\right\}$, $\operatorname{Var}(\psi)=\left\{y_{1}, \ldots, y_{m}\right\}$. By Definition $6 i_{h}(\chi)=\bigvee_{i \leq n} i_{h}\left(x_{i}\right)$ and $i_{h}(\psi)=$ $\bigvee_{j \leq m} i_{h}\left(y_{j}\right)$. As $\downarrow i_{h}(\chi)=\bigcup_{i \leq n} \downarrow i_{h}\left(x_{i}\right)=\mathfrak{s}_{\mathbf{A}}(\chi)$ and $\downarrow i_{h}(\psi)=\bigcup_{j \leq m} \downarrow$ $i_{h}\left(y_{i}\right)=\mathfrak{s}_{\mathbf{A}}(\psi)$, we obtain $\mathfrak{s}_{\mathbf{A}}(\chi) \subseteq \mathfrak{s}_{\mathbf{A}}(\psi)$ as desired. Conversely, the fact that 
$\mathfrak{s}_{\mathbf{A}}(\chi) \subseteq \mathfrak{s}_{\mathbf{A}}(\psi)$ implies $\downarrow i_{h}(\chi) \subseteq \downarrow i_{h}(\psi)$ and this, together with the fact that $i_{h}(\chi)=\bigvee_{i \leq n} i_{h}\left(x_{i}\right)$ and $i_{h}(\psi)=\bigvee_{j \leq m} i_{h}\left(y_{j}\right)$, entails $i_{h}(\chi) \leq i_{h}(\psi)$. This proves our claim.

Thus, $\mathfrak{v}_{\mathbf{A}}(\chi \rightarrow \psi)=1$ iff $h(\chi \rightarrow \psi) \in \bigcup_{i \in I}\left\{1^{\mathbf{A}_{i}}\right\}$, iff $i_{h}(\psi) \leq i_{h}(\chi)$ and (not both $h(\chi) \in \bigcup_{i \in I}\left\{1^{\mathbf{A}_{i}}\right\}$ and $\left.h(\psi) \notin \bigcup_{i \in I}\left\{1^{\mathbf{A}_{i}}\right\}\right)$, iff $\mathfrak{s}_{\mathbf{A}}(\psi) \subseteq \mathfrak{s}_{\mathbf{A}}(\chi)$ and (not both $\mathfrak{v}_{\mathbf{A}}(\chi)=1$ and $\mathfrak{v}_{\mathbf{A}}(\psi)=0$ ). So our claim is proved and $\mathfrak{M}_{\mathbf{A}}$ is a dependence model.

Since $1^{\mathbf{A}} \leq_{\vee}^{\mathbf{A}} h(\gamma)$ for all $\gamma \in \Gamma$, it follows that $\mathfrak{v}_{\mathbf{A}}(\gamma)=1$ for all $\gamma \in \Gamma$, whence $\mathfrak{v}_{\mathbf{A}}(\varphi)=1$ and so $h(x) \in \bigcup_{i \in I}\left\{1^{\mathbf{A}_{i}}\right\}$. Consequently, $1^{\mathbf{A}} \leq_{\vee}^{\mathbf{A}} h(\varphi)$.

Conversely, suppose that $\Gamma \nvdash_{\text {DAI }} \varphi$. Thus, there exists a dependence model $\mathfrak{M}=\langle\mathfrak{S}, \mathfrak{v}, \mathfrak{s}\rangle$ such that $\mathfrak{v}(\gamma)=1$ for all $\gamma \in \Gamma$, yet $\mathfrak{v}(\varphi)=0$. Consider the semilattice with zero $\langle\wp(\mathfrak{S}), \cup, \emptyset\rangle$, and let

$$
\mathfrak{T}^{m}=\left\langle\left\{\left\langle\mathbf{A}_{i}, F_{i}\right\rangle\right\}_{i \in \wp(\mathfrak{S})},\langle\wp(\mathfrak{S}), \cup, \emptyset\rangle,\left\{f_{i, j}: i, j \in \wp(\mathfrak{S}) \text { and } i \subseteq j\right\}\right\rangle,
$$

where each $\mathbf{A}_{i}$ is an isomorphic copy of $\mathbf{B}_{2}, F_{i}=\left\{1^{\mathbf{A}_{i}}\right\}$ for all $i \in \wp(\mathfrak{S})$, and $f_{i, j}(a)$ is the image of $a$ under the unique automorphism of $\mathbf{B}_{2}$. It can be checked that $\mathfrak{T}^{m}$ is a d-direct system of matrices. Now, define $h: \mathbf{F m}\left(\mathcal{L}_{1}\right) \rightarrow \mathbf{A} \mathbf{P}\left(\mathfrak{T}^{m}\right)$ as follows for any $\psi \in F m\left(\mathcal{L}_{1}\right): h(\psi)=1^{\mathbf{A}_{\mathfrak{s}(\psi)}}$ if $\mathfrak{v}(\psi)=1, h(\psi)=0^{\mathbf{A}_{\mathfrak{s}(\psi)}}$ otherwise. It follows that $1^{\mathbf{A}_{\mathbf{P}\left(\mathfrak{T}^{m}\right)}} \leq_{\leq_{\mathrm{P}}}^{\mathbf{A}_{\mathbf{P}\left(\mathfrak{T}^{m}\right)}} h(\gamma)$, for all $\gamma \in \Gamma$, while it is not the case that $1^{\mathbf{A}_{\mathbf{P}\left(\mathfrak{T}^{m}\right)}} \leq_{\vee}^{\mathbf{A}_{\mathbf{P}\left(\mathfrak{T}^{m}\right)}} h(\varphi)$.

\section{Leibniz Reduced Models}

In this section we characterise the Leibniz reduced models of the logic DAI. Recall that $\{\neg a \vee b, \neg b \vee a\}$ is a set of congruence formulas for CL, as the next lemma makes clear:

Lemma 27 [15, Ex. 6.67] Let $\langle\mathbf{A}, F\rangle \in \operatorname{Mod}^{*}(\mathrm{CL})$, and $a, b \in$ A. Then

$$
\langle a, b\rangle \in \Omega^{\mathbf{A}} F \Longleftrightarrow\{\neg a \vee b, \neg b \vee a\} \subseteq F .
$$

We will also need the following result.

Lemma 28 [15, Cor. 6.56] A set $\Delta(x, y)$ offormulas defines the Leibniz congruence of $F$ on $\mathbf{A}$ if and only if it defines the identity in $\left\langle\mathbf{A} / \Omega^{\mathbf{A}} F, F / \Omega^{\mathbf{A}} F\right\rangle$.

Theorem 29 Let $\langle\mathbf{A}, F\rangle \in \operatorname{Mod}(\mathrm{DAI})$. Then for all $a, b \in A,\langle a, b\rangle \in \Omega^{\mathbf{A}} F$ iff $\{a \rightarrow b, b \rightarrow a\} \subseteq F$.

Proof The left-to-right direction follows directly from Lemma 1. Conversely, let $a \rightarrow, b, b \rightarrow a \in F$. Suppose, towards a contradiction, that $\langle a, b\rangle \notin \Omega^{\mathbf{A}} F$, i.e. 
there exists a unary polynomial function $\varphi(x, \vec{v})$ such that for appropriate parameters $\vec{c} \in A, \varphi(a, \vec{c}) \in F$ iff $\varphi(b, \vec{c}) \notin F$. If we show the admissibility of the rule

$$
x \rightarrow y, y \rightarrow x, \varphi(x, \vec{v}) \vdash_{\mathrm{DAI}} \varphi(y, \vec{v})(\mathrm{R})
$$

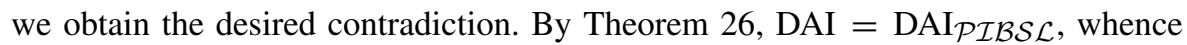
it suffices to show that the rule is admissible in the latter. To this end let $\langle\mathbf{C}, G\rangle \cong$ $\mathbf{P}\left(\mathfrak{T}^{m}\right) \in \operatorname{Mod}\left(\mathrm{DAI}_{\mathcal{P} \mathcal{I B S} \mathcal{L}}\right)$, where

$$
\mathfrak{T}^{m}=\left\langle\left\{\left\langle\mathbf{C}_{i}, G_{i}\right\rangle\right\}_{i \in I},\langle I, \leq, \perp\rangle,\left\{f_{i, j}: i, j \in I \text { and } i \leq j\right\}\right\rangle .
$$

W.l.o.g., we take $\mathbf{C}$ to be non trivial. Let $h: \mathbf{F m}\left(\mathcal{L}_{1}\right) \rightarrow \mathbf{C}$ be s.t. $h(x \rightarrow y), h(y \rightarrow$ $x), h(\varphi(x, \vec{v})) \in G$. At first observe that $h(x \rightarrow y), h(y \rightarrow x) \in G$ implies (i): $h(x), h(y) \in C_{i}$ and (ii): $h(x), h(y) \in G_{i}$ or $h(x), h(y) \in A_{i} \backslash G_{i}$. In fact, if (i) were false, $h(x \rightarrow y) \in G$ would imply $h(y \rightarrow x) \notin G$, while (ii) is justified by noticing that if $h(x) \in G$ and $h(y) \notin G$ we would have that $h(\neg x \vee y) \in G$ if and only if $h(\neg y \vee x) \notin G$.

By induction on the complexity of $\varphi(x, \vec{v})$ we show $h(\varphi(y, \vec{v})) \in G$. We assume that the variable $x$ actually occurs in $\varphi(x, \vec{v})$, for otherwise the rule $(\mathrm{R})$ is trivially admissible. For the base case, let first $\varphi(x, \vec{v})=x$; then our conclusion is immediate, as $h(x \rightarrow y), h(y \rightarrow x) \in G$ together with $h(x) \in G$ implies $h(y)=h(\varphi(y, \vec{v})) \in$ $G$. The cases $\varphi(x, \vec{v})=\neg x$ and $\varphi(x, \vec{v})=x * z$, for $* \in\{\wedge, \vee\}$, are also immediate (here $z$ is an arbitrary variable in $\vec{v}$ ). To round off the base case, let $\varphi(x, \vec{v})=x \rightarrow z$ (if $\varphi(x, \vec{v})=z \rightarrow x$ we argue analogously). As $h(\varphi(x, \vec{v}))=h(x) \rightarrow h(z) \in G$, we have $i_{h}(x) \leq i_{h}(z)$ and $h(x \vee z) \in G$, so $i_{h}(x)=i_{h}(y) \leq i_{h}(z)$. Moreover $h(\neg x) \vee h(z) \in G$ implies $h(x) \notin G$ or $h(z) \in G$. Recalling that if $h(x) \notin G$ then $h(y) \notin G$ we conclude $h(y \rightarrow z) \in G$. Next, we deal with the inductive step. If $\varphi(x, \vec{v})=\sigma(x, \vec{v}) * \epsilon(x, \vec{v})$, by induction hypothesis we can assume $h(\sigma(x, \vec{v})) \in G$ if and only if $h(\sigma(y, \vec{v})) \in G$ and $h(\epsilon(x, \vec{v})) \in G$ if and only if $h(\epsilon(y, \vec{v})) \in G$. This, together with the fact that $h(x), h(y)$ belong to the same fibre in $\mathbf{P}\left(\mathfrak{T}^{m}\right)$, entails $\varphi(y, \vec{v}) \in G$, proving the admissibility the rule $(\mathrm{R})$ in $\mathrm{DAI}_{\mathcal{P} \mathcal{I B S} \mathcal{L}}=\mathrm{DAI}$. This shows $\varphi(b, \vec{c}) \in G$, and leads us to the desired contradiction. So $\langle a, b\rangle \in \Omega^{\mathbf{A}} F$.

Theorem 29 boils down to the fact that $\{x \rightarrow y, y \rightarrow x\}$ is a set of congruence formulas for DAI. The next theorem identifies the Leibniz reduced models of DAI with a reduct representable as Płonka sum over a d-direct system of matrices.

Theorem 30 Let $\langle\mathbf{A}, F\rangle \in \operatorname{Mod}(\mathrm{DAI})$, such that its $\mathcal{L}_{0}$-reduct is a Płonka sum over a d-direct system

$$
\mathfrak{T}^{m}=\left\langle\left\{\left\langle\mathbf{A}_{i}, F_{i}\right\rangle\right\}_{i \in I},\langle I, \leq, \perp\rangle,\left\{f_{i, j}: i, j \in I \text { and } i \leq j\right\}\right\rangle .
$$

Then $\langle\mathbf{A}, F\rangle \in \operatorname{Mod}^{*}(\mathrm{DAI})$ iff either $\mathbf{A}$ is trivial, or for each $i \in I,\left\langle\mathbf{A}_{i}, F_{i}\right\rangle \in$ $\operatorname{Mod}^{*}(\mathrm{CL}) \backslash\left\{\left\langle\mathbf{B}_{1},\{1\}\right\rangle\right\}$.

Proof From left to right, consider $\langle\mathbf{A}, F\rangle \in \operatorname{Mod}^{*}(\mathrm{DAI})$. We show that for each $i \in I$ and for all $a, b \in A_{i}$ such that $a \neq b,\langle a, b\rangle \notin \Omega^{\mathbf{A}_{i}} F_{i}$. Fix $a, b \in A_{i}$. Since $\Omega^{\mathbf{A}} F=i d$, Theorem 29 implies that $\{a \rightarrow b, b \rightarrow a\} \nsubseteq F$. Moreover, as in this case $a \rightarrow b=\neg a \vee b$ and $b \rightarrow a=\neg b \vee a$, we obtain $\{\neg a \vee b, \neg b \vee a\} \nsubseteq F_{i}$ 
which, by Lemma 27 , entails $\langle a, b\rangle \notin \Omega^{\mathbf{A}_{i}} F_{i}$, as desired. It remains to show that if $\mathbf{A}$ is nontrivial, each fibre $\mathbf{A}_{i}$ is nontrivial. Suppose otherwise. This implies there exists $j \in I, j \neq i$ and, by Lemma 20 , for every $k \in I$ it holds $A_{k}=F_{k}$. Therefore $\Omega^{\mathbf{A}} F=A \times A$, a contradiction.

We now prove the other direction. Suppose either $\mathbf{A}$ is trivial, or for each $i \in I$, $\left\langle\mathbf{A}_{i}, F_{i}\right\rangle \in \operatorname{Mod}^{*}(\mathrm{CL}) \backslash\left\{\left\langle\mathbf{B}_{1},\{1\}\right\rangle\right\}$. If the former, then trivially $\Omega^{\mathbf{A}} F=i d$. So, let $\mathbf{A}$ be nontrivial, which entails that each fibre $\mathbf{A}_{i}$ is nontrivial. Fix $a, b \in A$ such that $a \neq b$; let moreover $a \in A_{i}$ and $b \in A_{j}$. Observe first that if $i=j$ then the assumption $\Omega^{\mathbf{A}_{i}} F_{i}=i d$ directly entails $\langle a, b\rangle \notin \Omega^{\mathbf{A}} F$. If $i \neq j$ consider $i \vee j=k$ (in case $i, j$ are comparable, assume w.l.o.g. $i<j$ ). Consider the unary polynomial function $x \rightarrow b$. Clearly $b \rightarrow b=1^{\mathbf{A}_{j}} \in F_{j} \subseteq F$, while $a \rightarrow b=0^{\mathbf{A}_{k}}$. The fact that $A_{i} \neq F_{i}$ and Lemma 20 imply $A_{k} \neq F_{k}$ and therefore $a \rightarrow b=0^{\mathbf{A}_{k}} \notin F_{k}$. By Lemma 1, we obtain $\langle a, b\rangle \notin \Omega^{\mathbf{A}} F$.

Corollary 31 Let $\langle\mathbf{A}, F\rangle \in \operatorname{Mod}(\mathrm{DAI})$, such that its $\mathcal{L}_{0}$-reduct is a Płonka sum over a d-direct system

$$
\mathfrak{T}^{m}=\left\langle\left\{\left\langle\mathbf{A}_{i}, F_{i}\right\rangle\right\}_{i \in I},\langle I, \leq, \perp\rangle,\left\{f_{i, j}: i, j \in I \text { and } i \leq j\right\}\right\rangle .
$$

Then $\Omega^{\mathbf{A}} F=\bigcup_{i \in i} \Omega^{\mathbf{A}_{i}} F_{i}$.

Proof Assume $\langle a, b\rangle \in \Omega^{\mathbf{A}} F$. By Theorem $29\{a \rightarrow b, b \rightarrow a\} \subseteq F$, which entails $a, b \in A_{i}$. So $\{\neg a \vee b, \neg b \vee a\} \in \Omega^{\mathbf{A}_{i}} F_{i} \subseteq \bigcup_{i \in i} \Omega^{\mathbf{A}_{i}} F_{i}$. The converse inclusion follows from Theorem 30 .

\section{Algebraisability and Related Properties}

In this final section, we show that DAI is algebraisable with $\mathcal{I I B S} \mathcal{L}$ as an equivalent algebraic semantics; moreover, we prove that it fails to be selfextensional. Thus, while DAI ranks among the best-behaved logics in terms of the so-called Leibniz hierarchy — which measures the "degree to which [logics] can be faithfully represented by the equational logic of [their algebraic counterpart]" [16, p. 44], it is not so ideal in terms of the Frege hierarchy, which measures the degree to which the interderivability relation of a given logic obeys by compositionality principles.

We start with a useful lemma. Throughout this section, given an involutive bisemilattice $\mathbf{A}$, we denote by $\mathbf{A} \rightarrow$ the result of adding to it a binary operation $\rightarrow$ according to the stipulations of Definition 21.

Lemma 32 Let $\mathbf{A} \in \mathcal{I I B S} \mathcal{L}$ and $\varphi, \psi \in F m\left(\mathcal{L}_{1}\right)$. For $h \in \operatorname{Hom}\left(\operatorname{Fm}\left(\mathcal{L}_{1}\right), \mathbf{A}\right)$, the following conditions are equivalent:

(1) $1 \leq_{\vee} h(\varphi \rightarrow \psi)=h(\varphi) \rightarrow^{\mathbf{A}} h(\psi)$;

(2) for every $v \in \operatorname{Hom}\left(\mathbf{F m}\left(\mathcal{L}_{1}\right),\left(\mathbf{A}^{-}\right) \rightarrow\right.$ such that $v(x)=h(x)$ for all $x \in$ $\operatorname{Var}\left(\mathcal{L}_{1}\right), 1 \leq_{\vee} v(\varphi \rightarrow \psi)=v(\varphi) \rightarrow\left(\mathbf{A}^{-}\right) \rightarrow v(\psi)$.

Proof W.l.o.g. we assume $\varphi, \psi$ do not contain occurrences of $\rightarrow$. 
(1) $\Rightarrow$ (2). Let $\mathbf{A}^{-} \cong \mathbf{P}(\mathfrak{T})$, where

$$
\mathfrak{T}=\left\langle\left\{\mathbf{A}_{i}\right\}_{i \in I},\langle I, \leq\rangle,\left\{f_{i, j}: i, j \in I \text { and } i \leq j\right\}\right\rangle .
$$

Recall that $\mathbf{A}^{-} \in \mathcal{I B S} \mathcal{L}$ and that $\mathbf{A}$ and $\left(\mathbf{A}^{-}\right) \rightarrow$ have the same $\rightarrow$-free reduct; therefore $v(\chi)=h(\chi)$ for every $\rightarrow$-free formula $\chi$ and for every $v: \mathbf{F m}\left(\mathcal{L}_{1}\right) \rightarrow\left(\mathbf{A}^{-}\right) \rightarrow$ that coincides with $h$ on the set $\operatorname{Var}\left(\mathcal{L}_{1}\right)$. Assume now $1 \leq_{\vee} h(\varphi \rightarrow \psi)=$ $h(\varphi) \rightarrow^{\mathbf{A}} h(\psi)$. By I3 in Definition 15 we have $i_{v}(\psi) \leq i_{v}(\varphi)$ and thus, by I2, $1 \leq_{\vee} v(\varphi \rightarrow \psi)=v(\neg \varphi \vee \psi)$, which, by Definition 21 entails $1 \leq_{\vee} v(\varphi \rightarrow \psi)=$ $v(\varphi) \rightarrow^{\left(\mathbf{A}^{-}\right)} \overrightarrow{ } v(\psi)$.

$(2) \Rightarrow(1)$. Let $v: \mathbf{F m}\left(\mathcal{L}_{1}\right) \rightarrow\left(\mathbf{A}^{-}\right) \rightarrow$ be such that $v(x)=h(x)$ for all $x \in$ $\operatorname{Var}\left(\mathcal{L}_{1}\right)$. Our assumption implies that $1 \leq_{\vee} v(\varphi \rightarrow \psi)=v(\varphi) \rightarrow^{\left(\mathbf{A}^{-}\right)} \rightarrow \overrightarrow{ } v(\psi)$. By Definition 21 we have $i_{v}(\psi) \leq i_{v}(\varphi)$ and $1 \leq_{\vee} v(\neg \varphi \vee \psi)$. Since $i_{v}(\psi) \leq i_{v}(\varphi)$ implies

$$
v(\varphi \wedge(\varphi \vee \psi))=h(\varphi \wedge(\varphi \vee \psi))=h(\varphi)=v(\varphi),
$$

by $\mathrm{I} 2$ in Definition 15 we obtain $1 \leq_{\vee} h(\varphi \rightarrow \psi)=h(\neg \varphi \vee \psi)=v(\neg \varphi \vee \psi)$, as desired.

Theorem 33 DAI is algebraisable with $\mathcal{I} \mathcal{I B S} \mathcal{L}$ as equivalent quasivariety semantics via the mappings $\tau(\alpha)=\{1 \vee \alpha \approx \alpha\}$ and $\rho(\alpha, \beta)=\{\alpha \rightarrow \beta, \beta \rightarrow$ $\alpha\}$.

Proof By Lemma 2, it will suffice to establish Conditions AL1 and AL4 in the Definition of algebraisability.

(AL1) We have to show that $\Gamma \vdash_{\text {DAI }} \varphi$ iff $\tau(\Gamma) \vdash_{E q(\mathcal{I} \mathcal{B S} \mathcal{L})} \tau(\varphi)$.

From left to right, assume $\Gamma \vdash_{\text {DAI }} \varphi$; w.l.o.g. $\Gamma=\left\{\gamma_{1}, \ldots, \gamma_{n}\right\}$ can be taken to be finite. Suppose towards a contradiction that there exist $\mathbf{A} \in \mathcal{I} \mathcal{I} \mathcal{B S}$ and $h \in \operatorname{Hom}\left(\mathbf{F m}\left(\mathcal{L}_{1}\right), \mathbf{A}\right)$ such that $1 \leq \vee h\left(\gamma_{1}\right), \ldots, h\left(\gamma_{n}\right)$, yet $1 \not \mathbb{}_{\vee} h(\varphi)$. By Lemma 32, for every $v \in \operatorname{Hom}\left(\mathbf{F m}\left(\mathcal{L}_{1}\right),\left(\mathbf{A}^{-}\right) \rightarrow\right)$ such that $v(x)=h(x)$ for all $x \in \operatorname{Var}\left(\mathcal{L}_{1}\right), 1 \leq_{\vee} v\left(\gamma_{1}\right), \ldots, v\left(\gamma_{n}\right)$, yet $1 \underline{\leq}_{\vee} v(\varphi)$. Since $\left(\mathbf{A}^{-}\right) \rightarrow \mathcal{P} \mathcal{I} \mathcal{B S} \mathcal{L}$, this means $\Gamma \nvdash_{\text {DAI }_{\mathcal{P I B S} \mathcal{L}}} \varphi$ and thus, by Theorem $26, \Gamma \nvdash_{\text {DAI }} \varphi$, which contradicts our assumption.

From right to left, since $\mathcal{P} \mathcal{I B S} \mathcal{L} \subseteq \mathcal{I} \mathcal{I B S} \mathcal{L}$, it follows that $\mathrm{DAI}_{\mathcal{I} \mathcal{I B S}} \subseteq$

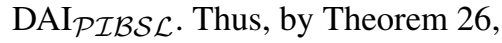

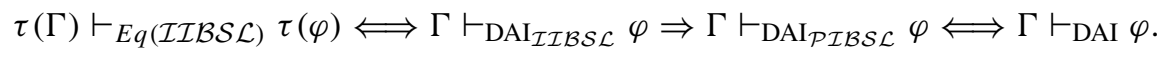

(AL4) We must prove that $\varphi \approx \psi \vdash_{E q(\mathcal{I} \mathcal{I B S} \mathcal{L})}\left\{1 \leq_{\vee} \varphi \rightarrow \psi, 1 \leq_{\vee} \psi \rightarrow \varphi\right\}$. Thus, let $\mathbf{A} \in \mathcal{I} \mathcal{I B S} \mathcal{L}$ and let $a \in A$. Recall that every $\mathcal{I B S L}$ (whence also $\mathbf{A}^{-}$) satisfies the identities $1 \leq \vee 1 \vee x \approx x \vee \neg x$. Since $a=a \wedge(a \vee a)$, by I2 in Definition 15 we have that $1 \leq \vee a \rightarrow a=a \vee \neg a$. Let now $a, b \in A$, and let $1 \leq_{\vee}^{\mathbf{A}} a \rightarrow b, 1 \leq_{\vee}^{\mathbf{A}} b \rightarrow a$. By I3, $a$ and $b$ belong to the same fibre $\mathbf{A}_{i}$ in the Płonka sum representation of $\mathbf{A}^{-}$. Thus, by I2, $1 \leq_{\vee}^{\mathbf{A}_{i}} b \vee \neg a, a \vee \neg b$, which implies (since $\mathbf{A}_{i}$ is Boolean) $a \leq_{\vee}^{\mathbf{A}_{i}} b \leq_{\vee}^{\mathbf{A}_{i}} a$, hence $a=b$.

To obtain an important corollary of algebraisability, we need a standard result in AAL. 
Theorem 34 [15, Prop. 4.57] Let L be algebraisable with equivalent quasivariety semantics $\mathcal{K}$ and with defining equations $\tau(x)$. Then $\langle\mathbf{A}, F\rangle \in \operatorname{Mod}^{*}(\mathrm{~L})$ if and only if $\mathbf{A} \in \mathcal{K}$ and $F=\left\{a \in A: \varphi^{\mathbf{A}}(a)=\psi^{\mathbf{A}}(a)\right.$, for all $\varphi(x) \approx \psi(x)$ in $\left.\tau(x)\right\}$.

The next corollaries refine Theorem 29 to a full characterisation of the Leibniz reduced models of DAI and their algebra reducts.

Corollary $35 \mathrm{Alg}^{*}(\mathrm{DAI})=\mathrm{A} \lg (\mathrm{DAI})=\mathcal{I} \mathcal{I} \mathcal{S} \mathcal{L}$.

Corollary $36\langle\mathbf{A}, F\rangle \in \operatorname{Mod}^{*}(\mathrm{DAI})$ if and only if $\mathbf{A} \in \mathcal{I} \mathcal{I B S} \mathcal{L}$ and $F=\{a \in A$ : $\left.1 \leq_{\vee}^{\mathbf{A}} a\right\}$.

Taking into account Theorems 22 and 26, Theorem 33 can be easily straightened up into a proof that $\mathcal{P} \mathcal{I B S} \mathcal{L}$ is an equivalent algebraic semantics of DAI as well. By Theorem 3, it follows that $\mathcal{I} \mathcal{I B S} \mathcal{L}=I S P P_{U}(\mathcal{P I B S} \mathcal{L})$. It is natural to wonder whether a stronger result holds to the effect that every implicative involutive bisemilattice is isomorphic to a P-implicative involutive bisemilattice, and whether all the matrices in Mod*(DAI) can be represented as Płonka sums over d-direct systems of matrices. The next example answers both questions in the negative.

Example 37 Consider the following algebra $\mathbf{A}$ of type $\mathcal{L}_{1}$, where the operations in the $\mathcal{L}_{0}$-reduct are computed according to Definition 7 , and $\rightarrow$ is defined as follows for $a, b \in A$ :

$$
a \rightarrow b=\left\{\begin{array}{l}
\neg a \vee b \text { if } a=a \wedge(a \vee b), \\
0 \text { otherwise. }
\end{array}\right.
$$

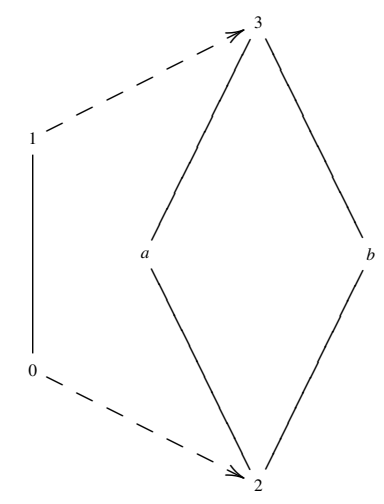

Clearly $\mathbf{A} \in \mathcal{I} \mathcal{I} \mathcal{B S} \mathcal{L}$, but $\mathbf{A} \notin \mathcal{P} \mathcal{I} \mathcal{B S} \mathcal{L}$, as

$$
0 \rightarrow^{\mathbf{A}} a=0 \text { while } 0 \rightarrow^{\left(\mathbf{A}^{-}\right) \rightarrow} a=3 .
$$

So, in particular, $\mathbf{A} \notin \mathcal{P} \mathcal{I} \mathcal{B S} \mathcal{L}$, and $\langle\mathbf{A},\{1,3\}\rangle$ is a Leibniz reduced model of DAI whose algebra reduct is not in $\left\{\mathbf{A}^{\rightarrow}: \mathbf{A} \in \mathcal{I} \mathcal{B S} \mathcal{L}\right\}$. 
We leave it for future research to determine whether $\mathcal{I} \mathcal{I B S} \mathcal{L}$ is a proper quasivariety, and to find out the exact relationships between the algebraic structures studied in this paper and the algebras investigated in [9].

Finally, we show that in terms of its placement within the Frege hierarchy, DAI is not even selfextensional.

\section{Theorem 38 DAI is not selfextensional.}

Proof We have to show that $\vdash_{\text {DAI }}$ is not a congruence on $\operatorname{Fm}\left(\mathcal{L}_{1}\right)$. To this end, it suffices to observe that $\alpha \vee \neg \alpha \dashv \vdash_{\text {DAI }} \beta \vee \neg \beta$ while $(\alpha \vee \neg \alpha) \rightarrow \alpha \nvdash_{\text {DAI }}$ $(\beta \vee \neg \beta) \rightarrow \alpha$.

Acknowledgments Preliminary versions of this paper were presented at the workshops Nonclassical modalities (Mexico City, September 2018) and Logic in Bochum IV (Bochum, October 2018). Thanks are due to the audiences of these talks for their insightful suggestions. A. Ledda and F. Paoli gratefully acknowledge the support of the Horizon 2020 program of the European Commission: SYSMICS project, number: 689176, MSCA-RISE-2015. All authors express their gratitude for the support of Fondazione di Sardegna within the project Science and its Logics: The Representation's Dilemma, Cagliari, CUP: F72 F16 003220 002, and the Regione Autonoma della Sardegna within the projects: Le proprietà d'ordine in matematica e fisica, CUP: F72 F16 002920 002, Per un'estensione semantica della Logica Computazionale Quantistica - Impatto teorico e ricadute implementative, RAS: SR40341.

Publisher's Note Springer Nature remains neutral with regard to jurisdictional claims in published maps and institutional affiliations.

\section{References}

1. Bonzio, S., Gil Férez, J., Paoli, F., Peruzzi, L. (2017). On paraconsistent weak Kleene logic: axiomatisation and algebraic analysis. Studia Logica, 105(2), 253-297.

2. Bonzio, S., Moraschini, T., Pra Baldi, M. Logics of left variable inclusion and Płonka sums of matrices, submitted for publication.

3. Burris S., \& Sankappanavar H.P. (2012). A course in universal algebra, the Millennium Edition. Available at https://www.math.uwaterloo.ca/ $\sim$ snburris/htdocs/ualg.html.

4. Cabrer, L., \& Priestley, H. (2015). A general framework for product representations: bilattices and beyond. Logic Journal of IGPL, 23, 816-841.

5. Carnielli, W. (1987). Methods of proof for relatedness and dependence logic. Reports on Mathematical Logic, 21, 35-46.

6. Ciuni, R., Ferguson, T.M., Szmuc, D. (2018). Relevant logics obeying component homogeneity. Australasian Journal of Logic, 15(2), 301-361.

7. Ciuni, R., Ferguson, T.M., Szmuc, D. Logics based on linear orders of contaminating values. typescript.

8. Dunn, J.M. (1972). A modification of Parry's analytic implication. Notre Dame Journal of Formal Logic, 13(2), 195-205.

9. Epstein, R.D. (1987). The algebra of dependence logic. Reports on Mathematical Logic, 21, 19-34.

10. Epstein R.D. (1995). The semantic foundations of logic: propositional logics, 2nd edn. New York: Oxford University Press.

11. Fariñas del Cerro, L., \& Lugardon, V. (1991). Sequents for dependence logic. Logique et Analyse, 34(133-134), 57-71.

12. Ferguson, T.M. (2014). A computational interpretation of conceptivism. Journal of Applied NonClassical Logics, 24(4), 333-367.

13. Ferguson, T.M. (2017). Meaning and proscription in formal logic. Berlin: Springer. 
14. Fine, K. (1986). Analytic implication. Notre Dame Journal of Formal Logic, 27(2), 169-179.

15. Font, J.M. (2016). Abstract algebraic logic: an introductory textbook. London: College Publications.

16. Font, J.M., Jansana, R., Pigozzi, D. (2003). A survey of abstract algebraic logic. Studia Logica, 74, 13-97.

17. Kielkopf, C.F. (1977). Formal sentential entailment. Washington: University Press of America.

18. Meyer, R.K. (1974). New axiomatics for relevant logics I. Journal of Philosophical Logic, 3, 53-86.

19. Parry, W.T. (1932). Implication. PhD Thesis, Harvard University.

20. Parry, W.T. (1968). The logic of C. I. Lewis. In Schilpp, P.A. (Ed.), The Philosophy of C. I. Lewis (pp. 115-154). London: Cambridge University Press.

21. Płonka J. (1967). On distributive quasilattices. Fundamenta Mathematicae, 60, 191-200.

22. Płonka J. (1967). On a method of construction of abstract algebras. Fundamenta Mathematicae, 61(2), 183-189.

23. Płonka J. (1968). Some remarks on direct systems of algebras. Fundamenta Mathematicae, 62(3), 301-308.

24. Romanowska, A. (1986). On regular and regularized varieties. Algebra Universalis, 23, $215-241$.

25. Romanowska, A., \& Smith, J. (2002). Modes. Singapore: World Scientific.

26. Sylvan, R. (2000). On reasoning: (Ponible) reason for (and also against) relevance. In Hyde, D., \& Priest, G. (Eds.), Sociative logics and their applications (pp. 175-188). Ashgate: Aldershot.

27. Urquhart, A. (1973). A semantical theory of analytic implication. Journal of Philosophical Logic, 2(2), 212-219. 